\title{
QUASILINEAR CONVEXITY AND QUASILINEAR STARS IN THE RAY SPACE OF A SUPERTROPICAL QUADRATIC FORM
}

\author{
ZUR IZHAKIAN AND MANFRED KNEBUSCH
}

\begin{abstract}
Relying on rays, we search for submodules of a module $V$ over a supertropical semiring on which a given anisotropic quadratic form is quasilinear. Rays are classes of a certain equivalence relation on $V$, that carry a notion of convexity, which is consistent with quasilinearity. A criterion for quasilinearity is specified by a Cauchy-Schwartz ratio which paves the way to a convex geometry on $\operatorname{Ray}(V)$, supported by a "supertropical trigonometry". Employing a (partial) quasiordering on Ray $(V)$, this approach allows for producing convex quasilinear sets of rays, as well as paths, containing a given quasilinear set in a systematic way. Minimal paths are endowed with a surprisingly rich combinatorial structure, delivered to the graph determined by pairs of quasilinear rays - apparently a fundamental object in the theory of supertropical quadratic forms.
\end{abstract}

\section{CONTENTS}

1. Introduction

2. Convex sets in the ray space

3. CS-ratios for anisotropic rays

4. Quasilinear sets and QL-stars

5. QL-enlargements

6. Maximal quasilinear sets

7. Convexity of QL-stars

8. Enlargements of QL-paths, and bridges to find short QL-paths

9. Interplay of the quasilinear ordering on the ray space with direct QL-paths

10. Minimal QL-paths and their anchor sets; the appearance of flocks

11. Further observations on minimal QL-paths and their anchor sets

12. Domination of minimal QL-paths

References

\section{INTRODUCTION}

This paper is a continuation of [5, 6], where quadratic forms on a free supertropical module were introduced and classified, as well as their bilinear companions, providing a

Date: May 24, 2022.

2010 Mathematics Subject Classification. Primary 15A03, 15A09, 15A15, 16Y60; Secondary 14T05, 15A33, 20M18, 51M20.

Key words and phrases. Tropical algebra, supertropical modules, bilinear forms, quadratic forms, quadratic pairs, ray spaces, convex sets, quasilinear sets, Cauchy-Schwartz ratio. 
tropical version of trigonometry. As the Cauchy-Schwarz inequality does not always hold on this setting, the so-called CS-ratio plays a major role in this theory. With this CS-ratio, for a suitable equivalence relation, whose equivalence classes are termed rays, a type of convex geometry on ray-spaces arises. The paper proceeds the study of this geometry.

In sequel to [4, 5, 6, 7, 8], our underlining structure is taken to be a supertropical semiring $R$ ([5, Definition 0.3] and [9, §3]); that is a semiring $R$ where $e:=1+1$ is idempotent (i.e., $e+e=e$ ) and, for all $x, y \in R, x+y \in\{x, y\}$ whenever $e x \neq e y$, otherwise $x+y=e y$. The ideal $e R$ of $R$ is a bipotent semiring (with unit element $e$ ), i.e., $u+v$ is either $u$ or $v$, for any $u, v \in e R$. The total ordering

$$
u \leq v \quad \Leftrightarrow \quad u+v=v
$$

of $e R$, together with the map $x \mapsto e x$, determines the addition of $R$ :

$$
x+y= \begin{cases}y & \text { if } e x<e y \\ x & \text { if } e x>e y \\ e y & \text { if } e x=e y\end{cases}
$$

Taking $y=0, e x=0 \Rightarrow x=0$. The elements of $\mathcal{T}(R):=R \backslash(e R)$ are called tangible, while those of $\mathcal{G}(R):=(e R) \backslash\{0\}$ are called ghost elements. The zero $0=e 0$ is regarded mainly as a ghost. $R$ itself is said to be tangible, if it is generated by $\mathcal{T}(R)$ as a semiring, namely iff $e \mathcal{T}(R)=\mathcal{G}(R)$. When $\mathcal{T}(R) \neq \emptyset$, discarding the "superfluous" ghost elements, $R^{\prime}:=\mathcal{T}(R) \cup e \mathcal{T}(R) \cup\{0\}$ is the largest tangible sub-semiring of $R$.

An $R$-module $V$ over a commutative semiring $R$ (with 1 ) is defined in the familiar way. A quadratic form on $V$ is a function $q: V \rightarrow R$ satisfying

$$
q(a x)=a^{2} q(x)
$$

for any $a \in R, x \in V$, such that

$$
q(x+y)=q(x)+q(y)+b(x, y)
$$

for any $x, y \in V$, where $b: V \times V \rightarrow R$ is a bilinear form, called a companion of $q$, not necessarily uniquely determined by $q$. The pair $(q, b)$ is called a quadratic pair on $V$.

A quadratic form $q$ with unique companion is called rigid. This is equivalent to $q\left(\varepsilon_{i}\right)=0$ for all $\varepsilon_{i}$ of a fixed base $\left\{\varepsilon_{i} \mid i \in I\right\}$ of $V$, by [5, Theorem 3.5]. $q$ is quasilinear, if $b=0$ is one of its companions, i.e., $q(x+y)=q(x)+q(y)$ for all $x, y \in V$. These are the "diagonal" forms

$$
q\left(\sum_{i} x_{i} \varepsilon_{i}\right)=\sum_{i} q\left(\varepsilon_{i}\right) x_{i}^{2}
$$

since $(\lambda+\mu)^{2}=\lambda^{2}+\mu^{2}$ for all $\lambda, \mu \in R$, cf. [5, Proposition 0.5].

Any quadratic form $q$ on a free $R$-module can be written as a sum

$$
q=q_{Q L}+\rho,
$$

where $q_{Q L}$ is a quasilinear (uniquely determined by $q$ ) and $\rho$ is rigid (but not unique), called the quasilinear part of $q$ and a rigid complement of $q_{\mathrm{QL}}$ in $q$, cf. [5, §4].

Aiming to detect on which parts of the underlying $R$-module $V$ a quadratic form is quasilinear, 6] studies the behavior of a quadratic pair $(q, b)$ on varying pairs of non-zero vectors 
$(x, y)$ in $V$, mostly for $R$ a tangible supersemifield or $e R$ a (bipotent) semifield. [6, Theorem 2.7] determines when a quadratic form is tangible or rigid.

A pair $(x, y)$ is called excessive, if $b(x, y)^{2}$ exceeds $q(x) q(y)$, in the sense [6. Definition 2.8], in particular when $q(x)=0$ or $q(y)=0$. However, by [6, Corollary 2.9], either $(x, y)$ is excessive or the restriction $q \mid R x+R y$ of $q$ is quasilinear. Nevertheless, this dichotomy does not depend on the companion $b$ of $q$, although $b$ takes part in defining excessiveness.

The CS-ratid 1 of anisotropic $x, y \in V$, i.e., $q(x) \neq 0, q(y) \neq 0$, is defined as

$$
\mathrm{CS}(x, y):=\frac{e b(x, y)^{2}}{e q(x) q(y)} \in e R .
$$

It serves as a tropical analogy to the familiar formula $\cos (x, y)=\frac{\langle x, y\rangle}{\|x\|\|y\|}$ in euclidian geometry, and leads to a version of "tropical trigonometry". (By squaring a formula there is no loss of information, since for any supertropical semiring the map $\lambda \mapsto \lambda^{2}$ is an injective endomorphism [5, Proposition 0.5].) For any anisotropic vector $w$, the function $x \mapsto \operatorname{CS}(x, w)$ is subadditive [6, Theorem 3.6].

A CS-ratio $\operatorname{CS}(x, y)$ can take values larger than $e=1_{e R}$, which does not happen in euclidian geometry; thereby features of noneuclidian geometry arise. These features are closely related to excessiveness, and are of main interest. When the set $e R$ is densely ordered, the pair $(x, y)$ is excessive iff $\operatorname{CS}(x, y)>e$. When $e R$ is discrete, $(x, y)$ is excessive if either $\mathrm{CS}(x, y)>c_{0}$, with $c_{0}$ the smallest element of $e R$ larger than $e$, or $\operatorname{CS}(x, y)=c_{0}$ and $q(x)$ or $q(y)$ is tangible. The pair $(x, y)$ is exotic quasilinear, if $\operatorname{CS}(x, y)=c_{0}$ and both $q(x)$ and $q(y)$ are ghost [6, Theorems 2.7 and 2.14]. This behavior bears relevance to problems of an arithmetical nature for classical quadratic forms, subject to tropicalization.

A projective version of the theory is obtained from the equivalence relation on $V \backslash\{0\}$ whose classes are called rays and defined as [6, §6]: Vectors $x, y$ in $V \backslash\{0\}$ belong to the same ray iff $\lambda x=\mu y$ for some $\lambda, \mu \in R \backslash\{0\}$. ( $\lambda, \mu$ need not be invertible as in the usual projective equivalence.) When $x$ and $y$ are anisotropic, $\operatorname{CS}(x, y)$ depends only on the rays $X, Y$ of $x, y$ and provides a well defined CS-ratio $\operatorname{CS}(X, Y)$ for anisotropic rays $X, Y$, i.e., rays $X, Y$ in $V \backslash q^{-1}(0)$. In terms of rays, subadditivity [6, Theorem 3.6] is better described by employing intervals $[X, Y]$, determined by rays $X, Y$. Given an anisotropic ray $Z \in[X, Y]$ and arbitrary $W,[6$, Theorem 7.7] compares $\operatorname{CS}(Z, W)$ with $\operatorname{CS}(X, W)+\operatorname{CS}(Y, W)$ while [6. Theorem 8.8] provides the uniqueness of the boundary of $[X, Y]$.

On the set $\operatorname{Ray}(V)$ of all rays, called the ray space of $V$, a natural notion of convexity appears: a subset $A \subset \operatorname{Ray}(V)$ is convex, if $[X, Y] \subset A$ for any $X, Y \in A$. With this notion, given a quadratic pair $(q, b)$, many problems of trigonometrical nature arise, some of which are addressed in this paper.

By studying the CS-ratio $\mathrm{CS}(X, Y)$ for $X \in S, Y \in T$ in given disjoint subsets $S, T$ of $\operatorname{Ray}(V)$, we obtain in $\$ 3$ rather subtle separation results for the convex hulls of $S$ and $T$ (in the obvious sense) from the Subadditivity Theorem [6, Theorem 3.6], compiled in Corollary 3.4 .

We call a pair $(X, Y)$ of rays in $V$ quasilinear (w.r. to $q$ ), if the restriction $q \mid R x+R y$ is quasilinear for any $x \in X, y \in Y$, and call a subset $C \subset \operatorname{Ray}(V)$ quasilinear, if any

\footnotetext{
1 "CS" is an acronym of "Cauchy-Schwarz".
} 
pairs $(X, Y)$ in $C$ are quasilinear. It turns our that the convex hull of $C$ is again quasilinear. In particular, if $C$ is a quasilinear subset of a convex set $A \subset \operatorname{Ray}(V)$, then the maximal quasilinear subsets of $A$ containing $C$ are convex. These objects are of central interest in $94-86$

The QL-star $\mathrm{QL}(X)$ of a ray $X$ (with respect to $q$ ) is the set of all $Y \in \operatorname{Ray}(V)$ for which the pair $(X, Y)$ is quasilinear, equivalently, the interval $[X, Y]$ is quasilinear. The QL-stars determine the quasilinear behavior of $q$ on the ray space. But, making this explicit, a major difficulty arises since a QL-star often is not quasilinear, and in some cases is not even convex, as explained in $\$ 4$ and $\$ 7$.

In $\$ 5$ we introduce a (partial) quasiordering on $\operatorname{Ray}(V)$, i.e., a transitive and reflexive relation $\preceq_{\mathrm{QL}}$, defined by

$$
X \preceq_{\mathrm{QL}} Y \quad \Leftrightarrow \quad \mathrm{QL}(X) \subset \mathrm{QL}(Y) .
$$

Given a quasilinear subset $C$ of $\operatorname{Ray}(V)$, this relation is employed to obtain new quasilinear sets containing $C$ in a systematic way. Namely, suppose $D$ is a subset of $\operatorname{Ray}(V)$ such that for every $Z \in D$ there is some $X \in C$ with $X \preceq_{\mathrm{QL}} Z$, then the convex hull $C^{\prime}$ of $C \cup D$ is again a quasilinear set, called a QL-enlargement of $C$. In particular we obtain a (unique) maximal QL-enlargement $E(C)$ of $C$ by taking $D$ to be the union of the up-sets $X^{\uparrow}=\left\{Z \in \operatorname{Ray}(V) \mid X \preceq_{\mathrm{QL}} Z\right\}$ with $X$ running through $C 2^{2}$

In 96 we study the family $\left(C_{i} \mid i \in I\right)$ of maximal quasilinear sets in $\operatorname{Ray}(V)$ containing a given quasilinear set $C$. We determine the union $\bigcup_{i \in I} C_{i}$ and the intersection $\widetilde{C}=\bigcap_{i \in I} C_{i}$ of these $C_{i}$, and prove that $C$ itself is maximal quasilinear (if and) only if $C$ is the intersection of the QL-stars of all rays in $C$. (One direction is trivial). I turns out that the maximal enlargement $E(C)$ is contained in $\widetilde{C}$.

It is a fundamental matter in the theory of supertropical quadratic forms to study the quasilinear graph $\Gamma_{\mathrm{QL}}(V, q)$ of $q: V \rightarrow R$, whose vertices and edges are respectively the rays and the quasilinear pairs of rays, in particular to describe the path components of $\Gamma_{\mathrm{QL}}(V, q)$ and to extract information about the diameters. Due to time and space limitation, we leave these topics to a future study, but in $\$ 8-\$ 12$ we provide a preparation for this.

In $₫ 8$ we define enlargements of a given QL-path $\left(X_{0}, \ldots, X_{n}\right)$ (i.e., a path in $\left.\Gamma_{\mathrm{QL}}(V, q)\right)$ by employing suitable QL-enlargements of the intervals $\left[X_{i}, X_{i+1}\right]$. We then develop in 99 a procedure for replacing $\left(X_{0}, \ldots, X_{n}\right)$ ba a shorter path from $X_{0}$ to $X_{n}$. This provides a rich interplay between QL-paths and the up-sets and down-sets of the quasiordering $\preceq_{\mathrm{QL}}$ on $\operatorname{Ray}(V)$.

We can retreat to the case that $T=\left(X_{0}, \ldots, X_{n}\right)$ is a direct QL-path, i.e., no pair $\left(X_{i}, X_{j}\right)$ in $T$ with $j>i+1$ is quasilinear, and then observe in $\oint 9$ that an upset $Y^{\uparrow}$ can meet $T$ in at most two rays $X_{p}, X_{q}$, which are then adjacent, $q=p \pm 1$ (Theorem 9.4). When this happens, we call $\left\{X_{p}, X_{q}\right\}$ a twin pair in $T$, and call $Y$ an anchor of $\left\{X_{p}, X_{q}\right\}$. A ray $X_{r}$ in $T$ which does not appear in a twin pair of $T$ is called a single of $T$. We regard any ray $Y$ with $X_{r} \in Y^{\uparrow}$ (i.e., $X_{r} \preceq{ }_{\mathrm{QL}} Y$ ) as an anchor of $Y$.

This gives us a sequence $S=\left(Y_{0}, \ldots Y_{m}\right)$, often not unique, which list anchors of all rays in $T$ in an economic way in the sense that there is a minimal monotonic surjective map

\footnotetext{
${ }^{2}$ For systematic reasons $X^{\uparrow}$ is denoted as sat ${ }_{\mathrm{QL}}$ in 95
} 
$\pi:[0, n] \rightarrow[0, m]$ such that some anchor of each $X_{i}$ is listed in $\left(Y_{0}, \ldots, Y_{\pi(i)}\right)$. (Necessarily $\pi(0)=0, \pi(n)=m$.) We call $S$ an anchor set of the QL-path $T$. It remains a widely open problem to find out which sequences $S$ appear as anchor sets of direct QL-paths, although we obtain some relevant information (cf. Theorem 9.10).

We are better off if $T$ is a minimal QL-path, i.e., a path of shortest length from $X_{0}$ to $X_{n}$. Given an anchor set $S$ of $T=\left(X_{0}, \ldots, X_{n}\right)$, we search in $\$ 10$ for partitions of $T$ into subpaths (which, of course, are again minimal) which together with their anchors in $S$ obey a simple and transparent combinatoric. Our main thrust is on subsequences $\left(X_{p}, \ldots, X_{q}\right)$, $q \geq p+2$, of $T$ such that any two adjacent rays $X_{p+i} X_{p+i+1}(0 \leq i<q-p)$ are twins in $T$. We call these subsequences flocks. Given an anchor set $S$ of $T$, we obtain in $\$ 12$ a modification $T^{\prime}$ of $T$, determined by $S$ in a unique way, which is again a QL-path from $X_{0}$ to $X_{n}$ of length $n$, and has a partition into maximal flocks, isolated twin pairs (i.e., twin pairs not appearing in a flock), and singles (i.e., rays not appearing in a twin pair). This partition of $T^{\prime}$ and the QL-path $T^{\prime}$ itself are uniquely determined by the anchor set $S$ of $T$. Then, surprisingly, it turns out that all this procedure does not depend on the choice of $S$, and so is determined by $T$ alone.

Here ample space is left for further study. In particular, it remains a mystery which sequences of maximal flocks, isolated twin pairs, and singles show up, while running through all minimal paths from $X_{0}$ to $X_{n}$.

Notation 1.1. $R^{*}$ denotes the group of units of a semiring $R$. In a supertropical semiring $R$

- $\mathcal{T}(R):=R \backslash e R$ is the set of tangible elements $\neq 0$,

- $\mathcal{G}(R):=e R \backslash\{0\}$ is the set of ghost elements $\neq 0$,

- $\nu_{R}$ denotes the ghost map $R \rightarrow e R, a \mapsto e a$.

When it is clear from the context, we write $\mathcal{T}, \mathcal{G}, \nu$ for $\mathcal{T}(R), \mathcal{G}(R), \nu_{R}$, and ea $=\nu(a)$ for $a \in R . a \leq_{\nu} b$ (resp. $a<_{\nu} b$ ) stands for ea $\leq$ eb (resp. ea $\left.<e b\right)$, the $\nu$-equivalence $a \cong_{\nu} b$ means that $e a=e b$.

\section{Convex SETS in The RAY SPACE}

We assume that $V$ is an $R$-module over a supertropical semiring $R$ whose ghost ideal $e R$ is a (bipotent) semifield. We compile some facts about convex sets and intervals in Ray $(V)$ without yet involving a quadratic form.

\section{Definition 2.1.}

(a) A subset $M$ of $\operatorname{Ray}(V)$ is convex (in $\operatorname{Ray}(V)$ ), if for any two rays $X, Y \in M$ the closed interval $[X, Y]$ is contained in $M$.

(b) The smallest convex subset of $\operatorname{Ray}(V)$ containing a nonempty set $S \subset \operatorname{Ray}(V)$ (which obviously exists) is called the convex hull of $S$ (in $\operatorname{Ray}(V)$ ) and is denoted by $\operatorname{conv}(S)$. When $S=\left\{X_{1}, \ldots, X_{n}\right\}$ is finite, we write $\operatorname{conv}(S)=\operatorname{conv}\left(X_{1}, \ldots, X_{n}\right)$, for short.

Example 2.2. For any rays $X, Y$ in $\operatorname{Ray}(V)$ all the intervals $] X, Y[] X, Y,],[X, Y[,[X, Y]$ (cf. [6, §6]) are convex sets [6, Proposition 8.1]. Clearly $[X, Y]=\operatorname{conv}(X, Y)$.

Proposition 2.3. 
(a) If $U_{1}, \ldots, U_{n}$ are ray-closed subsets of $V \backslash\{0\}$, i.e., unions of full rays, then the set $U_{1}+\cdots+U_{n}$ is again ray-closed in $V$, consisting of all rays $\operatorname{ray}_{V}\left(\lambda_{1} u_{1}+\cdots+\lambda_{n} u_{n}\right)$ with $u_{i} \in U_{i}, \lambda_{i} \in R \backslash\{0\}$. In particular, for any rays $X_{1}, \ldots, X_{n}$ in $V$ the set $X_{1}+\cdots+X_{n}$ is ray-closed in $V$.

(b) The convex hull of a finite set of rays $\left\{X_{1}, \ldots, X_{n}\right\}$ has the disjoint decomposition

$$
\operatorname{conv}\left(X_{1}, \ldots, X_{n}\right)=\bigcup_{i_{1}<\cdots<i_{r}} \operatorname{Ray}\left(X_{i_{1}}+\cdots+X_{i_{r}}\right)
$$

with $r \leq n$ and $1 \leq i_{1}<\cdots<i_{r} \leq n$.

Proof. The claims follow from the description of intervals in [6, Scholium 7.6] by an easy induction.

Notation 2.4. We denote by $\widetilde{A}$ the subset of $V \backslash\{0\}$, obtained as the union of all rays in a subset $A \subset \operatorname{Ray}(V)$. In other terms, $\widetilde{A}$ is the unique ray-closed subset of $V \backslash\{0\}$ with $\operatorname{ray}(\widetilde{A})=A$.

As the convex hull of a subset $A$ of $\operatorname{Ray}(V)$ is the union of all sets $\operatorname{conv}\left(X_{1}, \ldots, X_{r}\right)$ with $r \in \mathbb{N}, X_{1}, \ldots, X_{r} \in A$, we derive the following from Proposition 2.3 .(b).

Corollary 2.5. Assume that $A_{1}, \ldots, A_{n}$ are convex subsets of $\operatorname{Ray}(V)$. Let $C$ denote the convex hull of $A_{1} \cup \cdots \cup A_{n}$.

(a) $C$ is the union of all sets $\operatorname{conv}\left(X_{1}, \ldots, X_{n}\right)$ with $X_{i} \in A_{i}, 1 \leq i \leq n$.

(b) $\widetilde{C}$ is the union of all sets $\widetilde{A}_{i_{1}}+\cdots+\widetilde{A}_{i_{r}}$ with $r \leq n, 1 \leq i_{1}<\cdots<i_{r} \leq n$.

Proof. (a): Given $X_{1}, \ldots, X_{n}, Y_{1}, \ldots, Y_{n}$ with $X_{i}, Y_{i} \in A_{i}(1 \leq i \leq n)$ we have

$$
\begin{aligned}
\operatorname{conv}\left(\operatorname{conv}\left(X_{1}, \ldots, X_{n}\right), \operatorname{conv}\left(Y_{1}, \ldots, Y_{n}\right)\right) & =\operatorname{conv}\left(X_{1}, \ldots, X_{n}, Y_{1}, \ldots, Y_{n}\right) \\
& =\operatorname{conv}\left(\left[X_{1}, Y_{1}\right] \cup \cdots \cup\left[X_{n}, Y_{n}\right]\right) \\
& \subset \operatorname{conv}\left(A_{1} \cup \cdots \cup A_{n}\right),
\end{aligned}
$$

since $\left[X_{i}, Y_{i}\right]$ is the convex hull of $\left\{X_{i}, Y_{i}\right\}$.

(b): By Proposition 2.3. (b) for $X_{i} \in A_{i}(1 \leq i \leq n)$ we have

$$
\operatorname{conv}\left(X_{1}, \ldots, X_{n}\right)=\bigcup_{1 \leq i_{1}<\cdots<i_{r} \leq n} \operatorname{Ray}\left(X_{i_{1}}+\cdots+X_{i_{r}}\right) \subset \bigcup_{i_{1}<\cdots<i_{r}} \operatorname{Ray}\left(\widetilde{A}_{i_{1}}+\cdots+\widetilde{A}_{i_{r}}\right) .
$$

This together with part (a) implies the second claim.

Alternatively, Proposition 2.3 and Corollary 2.5 can be derived from the following observation, which deserves independent interest.

Remark 2.6. The convex subsets $A$ of $\operatorname{Ray}(V)$ correspond uniquely to the ray-closed submodules $W$ of $V$ via

$$
W=\widetilde{A} \cup\{0\}, \quad A=\operatorname{Ray}(W) .
$$

Proof. This is evident from the fact that for any $X, Y \in \operatorname{Ray}(V)$ the interval $[X, Y]$ is the set of all rays in the module $X_{0}+Y_{0}=(X+Y) \cup X \cup Y \cup\{0\}$, cf. [6, Remark 6.4]. 


\section{CS-RATios FOR ANisotropic RAYS}

We assume that $V$ is a module over a supertropical semiring $R$, whose ghost ideal $e R$ is a (bipotent) nontrivial semifield $3^{3}$, and that a quadratic pair $(q, b)$ on $V$ is given with $q$ anisotropic. In this situation, the $\mathrm{CS}$-ratio $\mathrm{CS}(X, Y)$ is well defined for any two rays $X, Y$ in the ray space $\operatorname{Ray}(V)$, such that

$$
\operatorname{CS}(X, Y)=\operatorname{CS}(x, y)=e \frac{b(x, y)^{2}}{q(x) q(y)}
$$

for any $x \in X, y \in Y$, cf. [6].

In what follows we exploit a major result from [6] on such CS-ratios, that is [6, Theorem 7.9], first from part (a) there, and later from parts (b) and (c).

Theorem 3.1. Given $\gamma \in e R$, let $S$ and $T$ be subsets of the ray space $\operatorname{Ray}(V)$ with $\mathrm{CS}(X, Y) \leq \gamma$ for all $X \in S$ and $Y \in T$. Then $\operatorname{CS}(Z, W) \leq \gamma$ for all $Z$ and $W$ in the convex hulls of $S$ and $T$ respectively.

Proof. a): We first prove that $\operatorname{CS}(Z, Y) \leq \gamma$ for $Z \in \operatorname{conv}(S), Y \in T$. By Proposition 2.3. (b) we have rays $X_{1}, \ldots, X_{n} \in S$ and vectors $x_{i} \in X_{i}(1 \leq i \leq n)$ such that

$$
Z=\operatorname{ray}\left(x_{1}+\cdots+x_{n}\right) \text {. }
$$

By [6, Theorem 7.9.a] there exist elements $\alpha_{1}, \ldots, \alpha_{n} \in \mathcal{G}$ with $\alpha_{1}+\cdots+\alpha_{n}=e$ such that

$$
\mathrm{CS}(Z, Y) \leq \sum_{i=1}^{n} \alpha_{i} \operatorname{CS}\left(X_{i}, Y\right)
$$

and so

$$
\mathrm{CS}(Z, Y) \leq \sum_{i=1}^{n} \alpha_{i} \gamma=\gamma
$$

b): Similarly, given a ray $Z \in \operatorname{conv}(S)$, we conclude that $\operatorname{CS}(Z, W) \leq \gamma$ for every $W \in$ $\operatorname{conv}(T)$.

\section{Remark 3.2.}

(a) In the same way, we see that if $\operatorname{CS}(X, Y)<\gamma$ for all $X \in S, Y \in T$, then $\operatorname{CS}(Z, W)<$ $\gamma$ for all $Z \in \operatorname{conv}(S), Y \in \operatorname{conv}(T)$. This can also be deduced from Theorem $2.1 \mathrm{in}$ a purely formal way, since every $Z \in \operatorname{conv}(S)$ is contained in the convex hull of some finite subset $S^{\prime}$ of $S$.

(b) When, in contrary to our present assumption, $q$ is not anisotropic, we restrict $q$ to the submodule

$$
V_{\text {an }}:=\{x \in V \mid q(x) \neq 0\} \cup\{0\}
$$

of $V$, and obtain the same result as above for subsets $S$ and $T$ in the ray space of $V_{\text {an }}$, which coincides with the convex subset $\operatorname{Ray}(V)_{\text {an }}$ of $\operatorname{Ray}(V)$ consisting of all anisotropic rays in $V$. Here it is important to note that the $R$-modules considered are not necessarily finitely generated, since even if $V$ is finitely generated, most often $V_{a n}$ is not.

\footnotetext{
${ }^{3}$ The term "nontrivial" means that $\mathcal{G} \neq\{e\}$.
} 
The proof of Theorem 3.1 was based on part (a) of [6, Theorem 7.9]. Next we exploit parts (b) and (c) of this theorem, which use the notion of $\nu$-quasilinearity of pairs of rays. We briefly recall this notion from [6], assuming that $e R$ is a nontrivial semifield.

A pair of vectors $x, y \in V$ is called $\nu$-quasilinear, if

$$
q(x+y) \cong_{\nu} q(x)+q(y),
$$

or equivalently, if the pair $(x, y)$ is quasilinear with respect to the quadratic form $e q$. A pair of rays $X, Y$ in $V$ is said to be $\nu$-quasilinear, if $(x, y)$ is $\nu$-quasilinear for all $x \in X$, $y \in Y$. When the rays $X, Y$ are anisotropic - a standard assumption in this section - the pair $(X, Y)$ turns out to be $\nu$-quasilinear iff $\operatorname{CS}(X, Y)<c$ for every $c>e$. In other terms, is the semifield $e R$ is dense, $\operatorname{CS}(X, Y) \leq e$, while, if $e R$ discrete, $\operatorname{CS}(X, Y) \leq c_{0}$ where $c_{0}$ is the smallest element of $e R$ that is bigger than $e$, cf. [6, Definition 7.3].

Theorem 3.3. Assume again that $e R$ is a nontrivial semifield and that $q$ is anisotropic. Let $S$ and $T$ be subsets of $\operatorname{Ray}(V)$ such that for any two rays $X \in S, Y \in T$ the pair $(X, Y)$ is $\nu$-quasilinear and $\mathrm{CS}(X, Y)$ is contained in a given convex subset $\Gamma$ of eR.

(a) Then every pair $(Z, W)$ with $Z \in \operatorname{conv}(S), W \in \operatorname{conv}(T)$ is $\nu$-quasilinear, and $\mathrm{CS}(Z, W) \in \Gamma$.

(b) If in addition all CS-ratios $\operatorname{CS}(X, Y)$ with $X \in S, Y \in T$ are contained in a fixed square class $\Delta$ of eR, cf. [5, Definition 7.1], then the same holds for all CS-ratios $\mathrm{CS}(Z, W)$ with $Z \in \operatorname{conv}(S), W \in \operatorname{conv}(T)$.

Proof. By assumption all CS-ratios $\mathrm{CS}(X, Y)$ with $X \in S, Y \in T$ are contained in the convex set $\Gamma \cap[0, e]$, if $e R$ is dense, or in convex set $\Gamma \cap\left[0, c_{0}\right]$, when $e R$ is discrete. Replacing $\Gamma$ by this smaller convex set, without loss of generality we may assume that $\Gamma \subset[0, e]$, respectively $\Gamma \subset\left[0, c_{0}\right]$.

To prove part (a) we follow the proof of Theorem 3.1. We first pick rays $Z \in \operatorname{conv}(S)$ and $Y \in T$, for which we have rays $X_{1}, \ldots, X_{n} \in S$ and vectors $x_{i} \in X_{i}$ such that $Z=$ $\operatorname{ray}\left(x_{1}+\cdots+x_{n}\right)$. By [6, Theorem 7.9.c] there exist $\alpha_{1}, \ldots, \alpha_{n}$ in $\mathcal{G}$ with $\alpha_{1}+\cdots+\alpha_{n}=e$ such that

$$
\mathrm{CS}(Z, Y)=\sum_{i=1}^{n} \alpha_{i} \mathrm{CS}(X, Y) .
$$

As the pairs $\left(X_{i}, Y\right)$ are $\nu$-quasilinear and all values $\operatorname{CS}\left(X_{i}, Y\right)$ are in $\Gamma$, also $\operatorname{CS}(Z, Y) \in \Gamma$, and so $(Z, Y)$ is $\nu$-quasilinear. Applying the same argument to a fixed $Z \in \operatorname{conv}(S)$ and varying $W \in \operatorname{conv}(T)$, we conclude that $\operatorname{CS}(Z, W) \in \Gamma$ for all $Z \in \operatorname{conv}(S), W \in \operatorname{conv}(T)$.

To prove part (b) we recall from [6, Remark 6.8] that for any ray $X$ in $V$ the set $e q(X)$ is a square class of the semifield $e R$. If $X$ and $Y$ are rays in $V$ and $x \in X, y \in Y$, then Formula (3.1) tells us that $\mathrm{CS}(X, Y) \in e q(X) \cdot e q(Y)$. Assume that all CS-ratios $\mathrm{CS}(X, Y)$ with $X \in S, Y \in T$ lie in a fixed square class $\Delta$ of $e R$. Given $Y \in T$, we conclude that

$$
e q(X)=\Delta \cdot q(Y)
$$

for all $X \in S$. Then [6, Theorem 7.9.b] applies for a given $Z \in \operatorname{conv}(S)$, and instead of (3.4) we obtain the relation

$$
\mathrm{CS}(Z, Y)=\sum_{i=1}^{n} \alpha_{i}^{2} \operatorname{CS}\left(X_{i}, Y\right)
$$


with $X_{i} \in S, \alpha_{i} \in e R, \alpha_{1}+\cdots+\alpha_{n}=e$. Namely, $\operatorname{CS}(Z, Y)$ is the maximum over the elements $\alpha_{i}^{2} \operatorname{CS}\left(X_{i}, Y\right)$ in $e R$, whence $\operatorname{CS}(Z, Y) \in \Delta$. Since this holds for all $Z \in \operatorname{conv}(S), Y \in T$, repeating the argument, we obtain that $\operatorname{CS}(Z, W) \in \Delta$ for all $Z \in \operatorname{conv}(S), W \in \operatorname{conv}(T)$, as claimed.

We draw the following consequences from Theorems 3.1 and 3.3 about disjointness of convex hulls in the ray space.

\section{Corollary 3.4.}

(a) Assume that the pair $(q, b)$ is balanced, i.e., $b(x, x)=e q(x)$ for all $x \in V$, cf. [5], §1] . Let $S$ and $T$ be subsets of $\operatorname{Ray}(V)$ with $\operatorname{CS}(X, Y)<e$ for all $X \in S, Y \in T$. Then the convex hulls of $S$ and $T$ are disjoint.

(b) Assume that e $R$ is discrete. Let $S$ and $T$ be subsets of $\operatorname{Ray}(V)$ with $\operatorname{CS}(X, Y)=c_{0}$ for all $X \in S, Y \in T$. Then again $\operatorname{conv}(S) \cap \operatorname{conv}(T)=\emptyset$.

(c) Let $(q, b)$ be balanced. Assume that all CS-ratios $\operatorname{CS}(X, Y)$ with $X \in S, Y \in T$ are contained in a fixed square class $\Delta \neq e R^{2}$, and furthermore that every pair $(X, Y)$ with $X \in S, Y \in T$ is $\nu$-quasilinear. Then again $\operatorname{conv}(S) \cap \operatorname{conv}(T)=\emptyset$.

Proof. a): It follows from Theorem 3.3.(a), applied with $\Gamma=[0, e[$, that $\operatorname{CS}(Z, W)<e$ for all $Z \in \operatorname{conv}(S), W \in \operatorname{conv}(T)$. But $\operatorname{CS}(Z, Z)=e$ for all $Z \in \operatorname{Ray}(V)$, and thus $\operatorname{conv}(S) \cap \operatorname{conv}(T)=\emptyset$. Alternatively, we obtain this result from Remark 3.2.(a), applied with $\gamma=e$.

b): We obtain from Theorem 3.3. (a) that $\operatorname{CS}(Z, W)=c_{0}$ for all $Z \in \operatorname{conv}(S), W \in \operatorname{conv}(T)$, while $\operatorname{CS}(Z, Z) \leq e$ for every $Z \in \operatorname{Ray}(V)$ (cf. [5, Eq. (1.7)]).

c): We know by Theorem 3.3.(b) that $\operatorname{CS}(Z, W) \in \Delta$ for any $Z \in \operatorname{conv}(S), W \in \operatorname{conv}(T)$, while $\operatorname{CS}(Z, Z)=e$ for every $Z \in \operatorname{Ray}(V)$.

\section{QuASILINEAR SETS AND QL-STARS}

We dismiss the assumption in 93 that $q$ is anisotropic, and first only assume that $V$ is an $R$-module over a supertropical semiring such that the pair $(R, V)$ is ray-admissible, i.e., for any $\lambda, \mu \in R$ and any $v \in V$

$$
\begin{array}{ll}
\lambda \neq 0, \mu \neq 0 & \Rightarrow \quad \lambda \mu \neq 0, \\
\lambda \neq 0, v \neq 0 & \Rightarrow \quad \lambda v \neq 0,
\end{array}
$$

so that the definition of rays in $V$ makes sense (cf. [6, §6]). We briefly say that the $R$ module $V$ is ray-admissible. (Note that (4.1) means that the semiring $R$ has no zerodivisors.)

We will exploit the following result, proved in [5, Proposition 1.20], which holds for any module $V$ over any semiring $R$.

\footnotetext{
${ }^{4}$ Recall from [5, §1] that every quadratic form on $V$ has a balanced companion.
} 
Theorem 4.1. Assume that $q: V \rightarrow R$ and $b: V \rightarrow R$ are a quadratic and a symmetric bilinear form on $V$, that $\left(x_{i} \mid i \in I\right)$ is a family of vectors in $V$, and that $b$ accompanies $q$ on the set $S:=\bigcup_{i \in I} R x_{i}$, i.e.,

$$
q(s+t)=q(s)+q(t)+b(s, t)
$$

for any $s, t \in S$. Then $b$ accompanies $q$ on the submodule $\sum_{i \in I} R x_{i}$ of $V$, generated by $S$.

Definition 4.2. Given a quadratic form $q$ on $V$, we call a (nonempty) subset $U$ of $\operatorname{Ray}(V)$ quasilinear (w.r. to $q$ ) if for any two rays $X, Y \in U$ the pair $(X, Y)$ is quasilinear, i.e.,

$$
q(x+y)=q(x)+q(y)
$$

for any two vectors $x \in X, y \in Y$.

Theorem 4.3. Assume that $q: V \rightarrow R$ is any quadratic form on the (ray-admissible) $R$ module $V$ and that $S$ is a quasilinear subset of $\operatorname{Ray}(V)$ (w.r. to q). Then the convex hull $\operatorname{conv}(S)$ is again quasilinear (w.r. to q).

Proof. Apply Theorem 3.1 with $b=0$.

Remark 4.4. We know that $\operatorname{conv}(X, Y)=[X, Y]$ for any two rays $X, Y$ in $V$. Theorem 4.3 tells us that, if the pair $(X, Y)$ is quasilinear, then the set $[X, Y]$ is quasilinear (in the sense of Definition 4.2). On the other hand, assuming that $R$ is supertropical with e $R$ a nontrivial semifield, in [6, §8], a closed interval $[X, Y]$ is defined to be quasilinear, if the pair $(X, Y)$ is quasilinean. But, as a consequence of Theorem 4.3, these notions of quasilinearity coincide for closed intervals.

We are ready for a key definition in this paper, assuming that the $R$-module $V$ is rayadmissible, where $R$ is any supertropical semiring.

Definition 4.5. The $Q L$-star $\mathrm{QL}(X)$ of a ray $X$ in $V$ is the set of all $Y \in \operatorname{Ray}(V)$ such that the pair $(X, Y)$ is quasilinear, equivalently, that the closed interval $[X, Y]$ is quasilinear.

It is evident from Definition 4.5, that

$$
X \in \mathrm{QL}(X)
$$

and, for any two rays $X, Y$ in $V$,

$$
X \in \mathrm{QL}(Y) \Leftrightarrow Y \in \mathrm{QL}(X) .
$$

Often $\mathrm{QL}(X)$ is not a quasilinear convex set (cf. Remark 4.7 below), and in rare cases $\mathrm{QL}(X)$ is not convex at all (cf. \$7 below). But we have the following useful fact.

Theorem 4.6. Assume that $X, Y, Z$ are rays in $V$ with $Y \in \mathrm{QL}(X), Z \in \mathrm{QL}(X)$ and that the pair $(Y, Z)$ is quasilinear. Then $[Y, Z] \subset \mathrm{QL}(X)$, and moreover

$$
\operatorname{conv}(X, Y, Z) \subset \mathrm{QL}(X) \cap \mathrm{QL}(Y) \cap \mathrm{QL}(Z) .
$$

\footnotetext{
${ }^{5}$ This makes sense since for a closed interval $I=[X, Y]$ the boundary rays $X, Y$ are uniquely determined by $I$ up to permutation [6, Theorem 7.9]
} 
Proof. All three intervals $[X, Y],[X, Z],[Y, Z]$ are quasilinear, and we conclude by Theorem 4.3 that the set $S=\operatorname{conv}(X, Y, Z)$ is also quasilinear. It follows that for any $W \in S$ the interval $[X, W]$ is contained in $S$, whence $S \subset \mathrm{QL}(X)$. Furthermore $[Y, Z] \subset \mathrm{QL}(X)$, since $S$ is quasilinear. By symmetry also $S \subset \mathrm{QL}(Y)$ and $S \subset \mathrm{QL}(Z)$, and so $S$ is contained in the intersection of the QL-stars of $X, Y, Z$.

Remark 4.7. Condition (4.6) is weaker than the condition that all three pairs $(X, Y)$, $(X, Z),(Y, Z)$ are quasilinear, since for three rays $X, Y, Z$ in $V$ with $\mathrm{QL}(X) \cap \mathrm{QL}(Y) \cap$ $\mathrm{QL}(Z) \neq \emptyset$ it may happen that $\operatorname{conv}(X, Y, Z)$ is not quasilinear. Take for example a free module $V$ of rank 4 with base $\left(\varepsilon_{i} \mid i \leq i \leq 4\right)$ over, say, a nontrivial tangible supersemifield $R$, and consider the quadratic form

$$
\left[\begin{array}{llll}
1 & \gamma & \gamma & 0 \\
& 1 & \gamma & 0 \\
& & 1 & 0 \\
& & & 1
\end{array}\right]
$$

for some $\gamma>_{\nu} 1$. Then the four basic rays $X_{i}=\operatorname{ray}\left(\varepsilon_{i}\right)$ have the property that $X_{4} \in \mathrm{QL}\left(X_{1}\right) \cap$ $\mathrm{QL}\left(X_{2}\right) \cap \mathrm{QL}\left(X_{3}\right)$, but $\operatorname{conv}\left(X_{1}, X_{2}, X_{3}\right)$ is not quasilinear. Note also that $\mathrm{QL}\left(X_{4}\right)=\operatorname{Ray}(V)$, so $\mathrm{QL}\left(X_{4}\right)$ is convex but, of course, not quasilinear.

We next analyze the situation that $\mathrm{QL}(X) \subset \mathrm{QL}\left(X^{\prime}\right)$ for given rays $X, X^{\prime}$ in $V$. As a preparation, we study intersections of QL-stars. For a nonempty subset $S$ of $\operatorname{Ray}(V)$ we set

$$
\mathrm{QL}(S):=\bigcap_{X \in S} \mathrm{QL}(X) .
$$

We read off from (4.4) that if $T$ is a second nonempty subset of $\operatorname{Ray}(V)$, then

$$
S \subset \mathrm{QL}(T) \Leftrightarrow T \subset \mathrm{QL}(S) .
$$

It may happen that $\mathrm{QL}(S)$ is empty, but otherwise the following holds.

Lemma 4.8. Assume that $\emptyset \neq S \subset \operatorname{Ray}(V)$ and $\mathrm{QL}(S) \neq \emptyset$.

(a) Then

$$
S \subset \mathrm{QL}(\mathrm{QL}(S))
$$

(b) If $S$ is quasilinear then

$$
S \subset \mathrm{QL}(S)
$$

Proof. (a): Given $X \in S$ we have $X \in \mathrm{QL}(Y)$ for every $Y \in \mathrm{QL}(S)$ (cf. (4.7)), and so $S \subset \mathrm{QL}(\mathrm{QL}(S))$.

(b): $S$ is contained in $\mathrm{QL}(X)$ for any $X \in S$, since $S$ is quasilinear, and so $S \subset \mathrm{QL}(S)$.

Lemma 4.9. If $S$ and $T$ are subsets of $\operatorname{Ray}(V)$ with both $\mathrm{QL}(S)$ and $\mathrm{QL}(T)$ nonempty, then

$$
\mathrm{QL}(S) \subset \mathrm{QL}(T) \quad \Leftrightarrow \quad T \subset \mathrm{QL}(\mathrm{QL}(S)) .
$$

Proof. QL $(S) \subset \mathrm{QL}(T) \Leftrightarrow \forall Y \in T: \mathrm{QL}(S) \subset \mathrm{QL}(Y) \Leftrightarrow \forall Y \in T: Y \in \mathrm{QL}(\mathrm{QL}(S))$ (cf. (4.7)) $\Leftrightarrow T \subset \mathrm{QL}(\mathrm{QL}(S))$.

Proposition 4.10. If $S \subset \operatorname{Ray}(V)$ and $\mathrm{QL}(S) \neq \emptyset$, then $\mathrm{QL}(\mathrm{QL}(S))$ is the biggest set $T \supset S$ in $\operatorname{Ray}(V)$ such that $\mathrm{QL}(S)=\mathrm{QL}(T)$. 
Proof. Let $T \supset S$. Then, of course, $\mathrm{QL}(T) \subset \mathrm{QL}(S)$ and thus $\mathrm{QL}(T)=\mathrm{QL}(S)$ iff $\mathrm{QL}(T) \supset$ $\mathrm{QL}(S)$, which by Lemma 4.9 occurs iff $T \subset \mathrm{QL}(\mathrm{QL}(S))$.

Supported by this proposition, we call the set QL $(\mathrm{QL}(S))$ the QL-saturum of $S$, provided that $\mathrm{QL}(S) \neq \emptyset$, and write

$$
\operatorname{sat}_{\mathrm{QL}}(\mathrm{S}):=\mathrm{QL}(\mathrm{QL}(\mathrm{S})) .
$$

We now turn to handle conveniently inclusion relations between QL-stars, focusing on the case $S=\{X\}$ for a single ray $X$ in $V$ in which $X \in \mathrm{QL}(S)$, and thus certainly $\mathrm{QL}(S) \neq \emptyset$. Writing

$$
\operatorname{sat}_{\mathrm{QL}}(\mathrm{X}):=\operatorname{sat}_{\mathrm{QL}}(\{\mathrm{X}\})=\mathrm{QL}(\mathrm{QL}(\mathrm{X}))
$$

we obtain

Theorem 4.11. If $X$ and $X^{\prime}$ are rays in $V$, then $\mathrm{QL}(X) \subset \mathrm{QL}\left(X^{\prime}\right)$ iff $X^{\prime} \in \operatorname{sat}_{\mathrm{QL}}(\mathrm{X})$.

Proof. A special case of Lemma 4.9,

\section{QL-ENLARGEMENTS}

We introduce a (partial) quasiordering $\preceq_{\mathrm{QL}}$ on $\operatorname{Ray}(V)$, i.e., a reflexive and transitive binary relation but not necessarily antisymmetric. For $X_{1}, X_{2} \in \operatorname{Ray}(V)$ we set

$$
X_{1} \preceq_{\mathrm{QL}} X_{2} \Leftrightarrow \mathrm{QL}\left(X_{1}\right) \subset \mathrm{QL}\left(X_{2}\right),
$$

which induces the equivalence relation:

$$
X_{1} \sim_{\mathrm{QL}} X_{2}: \Leftrightarrow X_{1} \preceq_{\mathrm{QL}} X_{2} \text { and } X_{2} \preceq_{\mathrm{QL}} X_{1} \Leftrightarrow \mathrm{QL}\left(X_{1}\right)=\mathrm{QL}\left(X_{2}\right) .
$$

Then, by Theorem 4.11 we obtain

$$
X_{1} \preceq_{\mathrm{QL}} X_{2} \Leftrightarrow X_{2} \in \operatorname{sat}_{\mathrm{QL}}\left(\mathrm{X}_{1}\right),
$$

and so

$$
X_{1} \sim_{\mathrm{QL}} X_{2} \Leftrightarrow X_{2} \in \operatorname{sat}_{\mathrm{QL}}\left(\mathrm{X}_{1}\right), \mathrm{X}_{1} \in \operatorname{sat}_{\mathrm{QL}}\left(\mathrm{X}_{2}\right)
$$

Using Lemma 4.9, we arrive at a third description of the quasiordering $\preceq_{\text {QL }}$, namely

$$
X_{1} \preceq_{\mathrm{QL}} X_{2} \Leftrightarrow \operatorname{sat}_{\mathrm{QL}}\left(\mathrm{X}_{2}\right) \subset \operatorname{sat}_{\mathrm{QL}}\left(\mathrm{X}_{1}\right),
$$

and so

$$
X_{1} \sim_{\mathrm{QL}} X_{2} \Leftrightarrow \operatorname{sat}_{\mathrm{QL}}\left(\mathrm{X}_{1}\right)=\operatorname{sat}_{\mathrm{QL}}\left(\mathrm{X}_{2}\right)
$$

Caution. In general it is not true that $\operatorname{sat}_{\mathrm{QL}}(\mathrm{X})$ is the set of all $Y \in \operatorname{Ray}(V)$ with $X \sim_{\mathrm{QL}} Y$.

Remark 5.1. Given a convex subset $A$ of $\operatorname{Ray}(V)$ we can establish the present theory for quasilinear subsets of $A$ instead of $\operatorname{Ray}(V)$ by defining for $X \in A$ the relative $Q L$-star

$$
\mathrm{QL}^{A}(X)=\mathrm{QL}(X) \cap A
$$

and defining on $A$ the quasioredering

$$
X \preceq_{\mathrm{QL}, A} Y \quad \Leftrightarrow \quad=\mathrm{QL}^{A}(X) \subset \mathrm{QL}^{A}(Y)
$$

with associated equivalence relation

$$
X \sim_{\mathrm{QL}, A} Y \quad \Leftrightarrow \quad=\mathrm{QL}^{A}(X)=\mathrm{QL}^{A}(Y) .
$$


But this is nothing really new, since the $R$-module $V$ can be replaced by the ray-closed $R$ submodule $W=\widetilde{A} \cup\{0\}$ defined in 9 .

This illustrates that it can be useful to work with quadratic pairs on a ray-admissible $R$-module instead of, say, just a free $R$-module.

The relation $\preceq_{\mathrm{QL}}$ allows to produce new quasilinear convex sets in $\operatorname{Ray}(V)$ from old ones. (All this holds for $R$ any supertropical semiring.)

Lemma 5.2. Let $X_{1}, X_{2}, X_{1}^{\prime}, X_{2}^{\prime}$ be rays in $V$ such that $X_{1} \preceq_{\mathrm{QL}} X_{1}^{\prime}, X_{2} \preceq_{\mathrm{QL}} X_{2}^{\prime}$, and the pair $\left(X_{1}, X_{2}\right)$ is quasilinear. Then $\operatorname{conv}\left(X_{1}, X_{2}, X_{1}^{\prime}, X_{2}^{\prime}\right)$ is a quasilinear convex set.

Proof. Since $X_{2} \in \mathrm{QL}\left(X_{1}\right)$ and $\mathrm{QL}\left(X_{1}\right) \subset \mathrm{QL}\left(X_{1}^{\prime}\right)$, also $X_{2} \in \mathrm{QL}\left(X_{1}^{\prime}\right)$, i.e., $\left(X_{1}^{\prime}, X_{2}\right)$ is quasilinear. Similarly, the pair $\left(X_{1}, X_{2}^{\prime}\right)$ is quasilinear. From $X_{1}^{\prime} \in \mathrm{QL}\left(X_{2}\right)$ we infer that $X_{1}^{\prime} \in$ $\mathrm{QL}\left(X_{2}^{\prime}\right)$, and so $\left(X_{1}^{\prime}, X_{2}^{\prime}\right)$ is quasilinear. Now Theorem 4.3 implies that $\operatorname{conv}\left(X_{1}, X_{2}, X_{1}^{\prime}, X_{2}^{\prime}\right)$ is quasilinear.

Theorem 5.3. Given a quasilinear convex subset $C$ of $\operatorname{Ray}(V)$, assume that $D$ is a subset of $\operatorname{Ray}(V)$ such that for every $Z \in D$ there exists some $X \in C$ with $X \preceq_{\mathrm{QL}} Z$. Then $\operatorname{conv}(D)$ is quasilinear.

Proof. Let $Z, W \in D$ be given, choose $X, Y \in C$ with $X \preceq_{\mathrm{QL}} Z, Y \preceq_{\mathrm{QL}} W$. Then $\operatorname{conv}(X, Y, Z, W)$ is a quasilinear convex set by Lemma 5.2. Thus, the pair $(Z, W)$ is quasilinear and hence $\operatorname{conv}(D)$ is quasilinear, by Theorem 4.3 .

Assume again that $C$ is a quasilinear convex subset of $\operatorname{Ray}(V)$ and $D$ is a subset of $\operatorname{Ray}(V)$ such that for every $Z \in D$ there is some $X \in C$ with $X \preceq_{\mathrm{QL}} Z$. Then we infer from Theorem 4.3 that

$$
C^{\prime}:=\operatorname{conv}(D \cup C)
$$

is a quasilinear convex set containing $C$.

Definition 5.4. A set $C^{\prime}$ of the form (5.9) is called an enlargement of the quasilinear convex set $C$. We also say that the pair $C \subset C^{\prime}$ is a quasilinear enlargement (or QLenlargement, for short). The set $D$ in (5.9) is said to be a mother set of $C^{\prime}$ (over $C$ ). Note that then $D \backslash C$ is also a mother set of $C^{\prime}$. A disjoint mother set of $C^{\prime}$ over $C$ is a mother set $D_{1}$ of $C^{\prime}$ with $D_{1} \cap C=\emptyset$.

The following is now obvious.

Remark 5.5. Let $C$ be a quasilinear convex (non-empty) subset of $\operatorname{Ray}(V)$.

a) The maximal mother set occurring for any enlargement of $C$ is

$$
D_{\infty}:=\left\{Z \in \operatorname{Ray}(V) \mid \exists X \in C: X \preceq_{\mathrm{QL}} Z\right\},
$$

in other terms (cf. (3.9)),

$$
D_{\infty}=\bigcup_{X \in C} \operatorname{sat}_{\mathrm{QL}}(\mathrm{X})
$$


b) The subsets of $D_{\infty}$ are precisely all mother sets of all enlargements of $C$, and

$$
E(C):=\operatorname{conv}\left(D_{\infty}\right)
$$

is the unique maximal enlargement of $C$.

Not every convex set $C^{\prime}$ with $C \subset C^{\prime} \subset E(C)$ is an enlargement of $C$. But, this is true when $C$ is a singleton $\left\{X_{0}\right\}$, as a consequence of the next theorem and Corollary 5.7 below.

Theorem 5.6. The $\mathrm{QL}-$ saturum $\operatorname{sat}_{\mathrm{QL}}\left(\mathrm{X}_{0}\right)$ of a ray $X_{0}$ in $V$ is a quasilinear convex subset of $\operatorname{Ray}(V)$.

Proof. Let $X_{1}, X_{2} \in \operatorname{sat}_{\mathrm{QL}}\left(\mathrm{X}_{0}\right)$ and $Z \in\left[X_{1}, X_{2}\right]$. We verify that $Z \in \operatorname{sat}_{\mathrm{QL}}\left(\mathrm{X}_{0}\right)$, which means that $\mathrm{QL}\left(X_{0}\right) \subset \mathrm{QL}(Z)$, cf. (5.1). Applying Theorem 5.3 to $D:=\left\{X_{1}, X_{2}\right\}$, we see that $\operatorname{conv}\left(X_{0}, X_{1}, X_{2}\right)$ is quasilinear. Given $W \in \mathrm{QL}\left(X_{0}\right)$, all pairs $\left(W, X_{i}\right), 0 \leq i \leq 2$, are quasilinear, since $\mathrm{QL}\left(X_{0}\right) \subset \mathrm{QL}\left(X_{i}\right)$. From Theorem 4.3 it follows that $\operatorname{conv}\left(X_{0}, X_{1}, X_{2}, W\right)$ is quasilinear, and then, that $[W, Z]$ is quasilinear. Thus $W \in \mathrm{QL}(Z)$, which proves that $\mathrm{QL}\left(X_{0}\right) \subset \mathrm{QL}(Z)$, as desired.

The following is now obvious.

Corollary 5.7. If $X$ is a ray in $V$, then $\operatorname{sat}_{\mathrm{QL}}(\mathrm{X})$ is the maximal enlargement of the quasilinear convex set $\{X\}$. The convex sets $C \subset \operatorname{sat}_{\mathrm{QL}}(\mathrm{X})$ with $X \in C$ are precisely all enlargements of $\{X\}$.

The QL-enlargements $\{X\} \subset C$ with $C$ convex in $\operatorname{sat}_{\mathrm{QL}}(\mathrm{X})$ may be regarded as the "atoms" (or perhaps better "molecules") in the set of all enlargements in the ray space $\operatorname{Ray}(V)$. To elaborate this view we introduce the concept of "amalgamating" a family of enlargements.

Proposition 5.8. Let $\left(C_{i 0} \subset C_{i} \mid i \in I\right)$ be a family of QL-enlargements in $\operatorname{Ray}(V)$. Define

$$
C_{0}:=\operatorname{conv}\left(\bigcup_{i \in I} C_{i 0}\right), \quad C:=\operatorname{conv}\left(\bigcup_{i \in I} C_{i}\right) .
$$

Then $C_{0} \subset C$ is again a QL-enlargement. If $D_{i}$ is a mother set of $C_{i}$ over $C_{i 0}$, then $D:=\bigcup_{i \in I} D_{i}$ is a mother set of $C$ over $C_{0}$.

Proof. We have a chain of equalities of convex hulls:

$$
\operatorname{conv}\left(D \cup C_{0}\right)=\operatorname{conv}\left(D \cup \bigcup_{i} C_{i 0}\right)=\operatorname{conv}\left(\bigcup_{i}\left(D_{i} \cup C_{i 0}\right)\right)=\operatorname{conv}\left(\bigcup_{i} C_{i}\right)=C .
$$

Furthermore, for a given ray $Z \in D$, there exists some $i \in I$ with $Z \in D_{i}$, and so some ray $X \preceq{ }_{\mathrm{QL}} Z$ with $X \in C_{i 0}$. Thus $X \in C_{0}$.

\section{Definition 5.9.}

a) Given a family $\left(C_{i 0} \subset C_{i} \mid i \in I\right)$ of QL-enlargements in $\operatorname{Ray}(V)$, we call the enlargement $C_{0} \subset C$ obtained in Proposition 5.8, cf. (5.12), the amalgamation of this family. The amalgamation $C_{0} \subset C$ is called special, if $C=\bigcup_{i \in I} C_{i}$ (instead of $\left.C=\operatorname{conv}\left(\bigcup_{i} C_{i}\right)\right)$, and very special, if in addition $C_{0}=\bigcup_{i \in I} C_{0 i}$. 
b) We call a QL-enlargement $C_{0} \subset C$ atomic, if $C_{0}$ is one point set $\{X\}$ in $\operatorname{Ray}(V)$. Note that then $C$ is a convex subset of $\operatorname{sat}_{\mathrm{QL}}(\mathrm{X})$ containing $X$ (Corollary 5.7).

Special amalgamations of families of atomic QL-enlargements arise naturally as follows.

Scholium 5.10. Let $C$ be a quasilinear convex set in $\operatorname{Ray}(V)$. Choose a family $\left\{\operatorname{sat}_{\mathrm{QL}}\left(\mathrm{X}_{\mathrm{i}}\right) \mid \mathrm{i} \in \mathrm{I}\right\}$ of QL-saturations of rays $X_{i} \in C$ which covers the set $C$, i.e.,

$$
C \subset \bigcup_{i \in I} \operatorname{sat}_{\mathrm{QL}}\left(\mathrm{X}_{\mathrm{i}}\right)
$$

Let $C_{i}:=C \cap \operatorname{sat}_{\mathrm{QL}}\left(\mathrm{X}_{\mathrm{i}}\right)$ and $C_{0}:=\operatorname{conv}\left(X_{i} \mid i \in I\right)$. Then $C_{0} \subset C$ is a special amalgamation of the family $\left(\left\{X_{i}\right\} \subset C_{i} \mid i \in I\right)$.

Families of atomic enlargements are of help to produce QL-enlargements $C_{0} \subset C$ for a quasilinear convex set $C$ with $C_{0}$ "small". Note that for inclusions $C_{0} \subset C_{1} \subset C$ of quasilinear convex sets, where $C_{0} \subset C$ is a QL-enlargement, the inclusion $C_{1} \subset C$ is an QLenlargement. We say that the QL-enlargement $C_{0} \subset C$ encompasses the QL-enlargement $C_{1} \subset C$.

Construction 5.11. Given a QL-enlargement $C_{1} \subset C$, we aim for a family of atomic QL-enlargements whose amalgamation encompasses $C_{1} \subset C$. We first choose a mother set $D \supset C_{1}$ of $C$ and a subset $S \subset C_{1}$ such that for every $Z \in D$ there is some $X \in S$ with $X \preceq_{\mathrm{QL}} Z$. Taking a labeling $S=\left\{X_{i} \mid i \in I\right\}$ of $S$, we define $E_{i}:=C \cap \operatorname{sat}_{\mathrm{QL}}\left(\mathrm{X}_{\mathrm{i}}\right)$. Clearly $D \subset \bigcup_{i \in I} E_{i}$, and so

$$
C=\operatorname{conv}(D)=\operatorname{conv}\left(\bigcup_{i \in I} E_{i}\right) .
$$

Furthermore $C_{0}=\operatorname{conv}\left(X_{i} \mid i \in I\right)$ for $C_{0}:=\operatorname{conv}(S) \subset C_{1}$. Thus $C_{0} \subset C$ is the amalgamation of the family $\left(\left\{X_{i}\right\} \subset E_{i} \mid i \in I\right)$, and $C_{0} \subset C_{1}$.

\section{Maximal quasilinear sets}

Given a nonempty quasilinear subset $S$ of $\operatorname{Ray}(V)$, by Zorn's Lemma there exists a maximal quasilinear set $C^{\prime} \supset S$ in $\operatorname{Ray}(V)$, because the union of a chain of quasilinear sets obviously is again quasilinear. Since the convex hull of any quasilinear set $S$ is again quasilinear (Theorem 4.3), the maximal quasilinear set $C^{\prime}$ is convex and moreover $C^{\prime} \supset \operatorname{conv}(S)$. Thus in the search for maximal quasilinear sets containing $S$ we may assume from the beginning that $S$ is convex.However, many of the formal arguments below remain valid without this convexity assumption.

In what follows $C$ denotes a fixed nonempty quasilinear subset of $\operatorname{Ray}(V)$ and $\left(C_{i} \mid i \in I\right)$ denotes the set of all maximal quasilinear sets of $\operatorname{Ray}(V)$ containing $C$.

Theorem 6.1. $\mathrm{QL}(C)=\bigcup_{i \in I} C_{i}$.

Proof. Since every $C_{i}$ is quasilinear and $C_{i} \supset C$, it is obvious that $C_{i} \subset \mathrm{QL}(C)$. If $X \in$ $\mathrm{QL}(C)$ is given, then for every $Y \in C$ the pair $(X, Y)$ is quasilinear. Thus the set $C \cup\{X\}$ is quasilinear, directly by Definition 3.2, and so $X \in C_{i}$ for some $i \in I$. 
Corollary 6.2. $C$ is maximal quasilinear iff $\mathrm{QL}(C)=C$.

Proof. This is the case $|I|=1$ of the theorem.

We define

$$
\widetilde{C}:=\bigcap_{i \in I} C_{i}
$$

This is the maximal quasilinear set containing $C$, such that $\left(C_{i} \mid i \in I\right)$ as well is the family of all maximal quasilinear sets containing $\widetilde{C}$.

We denote by $\operatorname{Max}(C)$ the set of maximal quasilinear subsets of $\operatorname{Ray}(V)$ which contain $C$, assuming tacitly that $C \neq \emptyset$. Thus, in the above notation,

$$
\operatorname{Max}(C):=\left(C_{i} \mid i \in I\right) .
$$

We have just observed that

$$
\operatorname{Max}(C)=\operatorname{Max}(\widetilde{C}) .
$$

As seen by Theorem 6.1, $\mathrm{QL}(C)$ is the union of all $E \in \operatorname{Max}(C)$, and thus (6.3) implies that

$$
\mathrm{QL}(C)=\mathrm{QL}(\widetilde{C})
$$

for any (nonempty) quasilinear subset $C$ of $\operatorname{Ray}(V)$.

Theorem 6.3. For every quasilinear subset $C$ of $\operatorname{Ray}(V)$

$$
\mathrm{QL}(\mathrm{QL}(C))=\mathrm{QL}(\mathrm{QL}(\widetilde{C}))=\widetilde{C} .
$$

Proof. We conclude from Theorem 6.1 and Corollary 6.2 that

$$
\mathrm{QL}(\mathrm{QL}(C))=\mathrm{QL}\left(\bigcup_{i \in I} C_{i}\right)=\bigcap_{i \in I} \mathrm{QL}\left(C_{i}\right)=\bigcap_{i \in I} C_{i}=\widetilde{C} .
$$

Furthermore $\mathrm{QL}(\widetilde{C})=\mathrm{QL}(C)$ by (‥4) and so $\mathrm{QL}(\mathrm{QL}(\widetilde{C}))=\widetilde{C}$.

The set $\mathrm{QL}(\mathrm{QL}(C))$ had been named the QL-saturum of $C$ in $\$ 4$. Consequently, we say that $C$ is QL-saturated if $C=\mathrm{QL}(\mathrm{QL}(C))$, i.e., $C=\widetilde{C}$. That is, in notation (4.10),

$$
\widetilde{C}=\operatorname{sat}_{\mathrm{QL}}(\mathrm{C})
$$

for every quasilinear subset $C$ of $\operatorname{Ray}(V)$. Clearly, the QL-saturum of $C$ is convex.

Theorem 6.4. Given nonempty quasilinear subsets $C$ and $D$ of $\operatorname{Ray}(V)$, the following assertions are equivalent.

(i) $\widetilde{C} \subset \widetilde{D}$,

(ii) $\operatorname{Max}(D) \subset \operatorname{Max}(C)$,

(iii) $\mathrm{QL}(D) \subset \mathrm{QL}(C)$.

Proof. (ii) $\Rightarrow$ (i): Evident, since $\widetilde{C}$ and $\widetilde{D}$ are the intersections of the families $\operatorname{Max}(C)$ and $\operatorname{Max}(D)$, respectively.

(i) $\Rightarrow$ (ii): $\widetilde{C} \subset \widetilde{D}$ implies that $\operatorname{Max}(\widetilde{C}) \supset \operatorname{Max}(\widetilde{D})$ directly by the definition of $\operatorname{Max}(\widetilde{C})$ and $\operatorname{Max}(\widetilde{D})($ cf. (6.2) $)$. This means $\operatorname{Max}(C) \supset \operatorname{Max}(D)$ by (6.3).

(ii) $\Rightarrow$ (iii): Immediate, since $\mathrm{QL}(C)$ and $\mathrm{QL}(D)$ are the unions of the families of sets $\operatorname{Max}(C)$ and $\operatorname{Max}(D)$ (Theorem 6.1). 
(iii) $\Rightarrow$ (ii): Given a maximal quasilinear set $E$ in $\operatorname{Ray}(V)$ we have the following chain of implications: $E \in \operatorname{Max}(D) \Leftrightarrow D \subset E \Rightarrow E=\mathrm{QL}(E) \subset \mathrm{QL}(D) \underset{\text { (iii) }}{\Longrightarrow} \subset \mathrm{QL}(C) \Rightarrow$ $\widetilde{C}=\mathrm{QL}(\mathrm{QL}(C)) \subset \mathrm{QL}(E)=E \Rightarrow E \in \operatorname{Max}(\widetilde{C})=\operatorname{Max}(C)$. This proves that $\operatorname{Max}(D) \subset$ $\operatorname{Max}(C)$.

We state a consequence of this theorem for the maximal QL-enlargement $E(C)$ of a convex quasilinear set $C$ in $\operatorname{Ray}(V)$.

Corollary 6.5. $E(C) \subset \widetilde{C}$, and $E(\widetilde{C})=\widetilde{C}$, i.e., $\widetilde{C}$ has no proper QL-enlargements.

Proof. $E(C)$ is the convex hull of the union of the sets $\operatorname{sat}_{\mathrm{QL}}(\mathrm{X})=\widetilde{\{\mathrm{X}\}}$ with $X$ running through $C$ (cf. Remark [5.5). Since by Theorem $6.4 \widetilde{\{X\}} \subset \widetilde{C}$ for each $X \in C$, we conclude that $E(C) \subset \widetilde{C}$. It follows that $E(\widetilde{C})=(\widetilde{C})^{\sim}=\widetilde{C}$.

\section{Convexity of QL-STARS}

We return to the assumptions made in $\$ 3$. To repeat, $V$ is a module over a supertropical semiring $R$, whose ghost ideal $e R$ is a nontrivial semifield, and $(q, b)$ is a quadratic pair on $V$ with $q$ anisotropic. Thus, for any two rays $X, Y$ in $V$ we have a well defined CS-ratio $\mathrm{CS}(X, Y)$, such that

$$
\operatorname{CS}(X, Y)=\operatorname{CS}(x, y)=\frac{e b(x, y)^{2}}{e q(x) q(y)}
$$

for $x \in X, y \in Y$. In addition, we assume that $e \mathcal{T}=\mathcal{G}$, but we do not require that every element of $\mathcal{T}$ is a unit in $R$, which would mean that $R$ is a tangible supersemifield.

We ask, in which cases a given QL-star $\mathrm{QL}(X)$ is convex in $\operatorname{Ray}(V)$. There is no serious problem if $e R$ is a dense semifield.

Theorem 7.1. If $e R$ is a dense semifield, then $\mathrm{QL}(X)$ is convex for every $X \in \operatorname{Ray}(V)$.

Proof. It follows from [6, Theorem 6.7] that now $\mathrm{QL}(X)$ is the set of all $Y \in \operatorname{Ray}(V)$ with $\operatorname{CS}(X, Y) \leq e$. If $Y_{1}, Y_{2}$ are rays in $V$ with $\operatorname{CS}\left(X, Y_{1}\right) \leq e, \operatorname{CS}\left(X, Y_{2}\right) \leq e$, then for every $Z \in[X, Y]$ also $\operatorname{CS}(X, Z) \leq e$ due to [6, Theorem 7.7.a] (a special case of the subadditivity theorem [6, Theorem 3.6.a]). Thus $\left[Y_{1}, Y_{2}\right] \subset \mathrm{QL}(X)$.

We turn to the case that the nontrivial semifield $e R$ is discrete. Then the totally ordered set $e R$ contains a smallest element $c_{0}>e R$, and, as known from [6, Theorem 6.7], a pair of rays $(X, Y)$ is quasilinear, if either $\operatorname{CS}(X, Y) \leq e$, or $\operatorname{CS}(X, Y)=c_{0}$ and both rays $X, Y$ are $g$-isotropic, i.e., $q(X)$ and $q(Y)$ are subsets of $\mathcal{G}$. In the latter case the pair $(X, Y)$ is called exotic quasilinear [6, Definition 6.6].

Theorem 7.2. The QL-stars of all $g$-anisotropic rays in $V$ are convex.

Proof. If $X$ is an $g$-anisotropic ray in $V$, then there does not exist $Y \in \operatorname{Ray}(V)$ such that the pair $(X, Y)$ is exotic quaislinear. Thus

$$
\mathrm{QL}(X)=\{Y \in \operatorname{Ray}(V) \mid \operatorname{CS}(X, Y) \leq e\} .
$$

We conclude as in the proof of Theorem 7.1 that $\mathrm{QL}(X)$ is convex.

If $X$ is $g$-isotropic, it may happen that $\mathrm{QL}(X)$ is not convex. 
Example 7.3. Assume that $\varepsilon_{1}, \varepsilon_{2}, \varepsilon_{3}$ are rays in $V$ with $q\left(\varepsilon_{i}\right)=e, b\left(\varepsilon_{i}, \varepsilon_{j}\right)=\gamma \in \mathcal{T}$ with e $\gamma=c_{0} .(1 \leq i<j \leq 3)$. (Note that this situation can be easily realised for $V$ a free module with base $\varepsilon_{1}, \varepsilon_{2}, \varepsilon_{3}$.) Let $X_{i}:=\operatorname{ray}\left(\varepsilon_{i}\right), Y_{i}:=\operatorname{ray}\left(\varepsilon_{j}+\varepsilon_{k}\right)$, where $i, j, k$ is a permutation of $\{1,2,3\}$. Then $Y_{i} \in\left[X_{j}, X_{k}\right]$. We compute

$$
\begin{array}{ll}
q\left(\varepsilon_{j}+\varepsilon_{k}\right)=e+e+\gamma=\gamma, & b\left(\varepsilon_{i}, \varepsilon_{j}+\varepsilon_{k}\right)=\gamma+\gamma=c_{0}, \\
\operatorname{CS}\left(X_{i}, Y_{i}\right)=\frac{e \gamma^{2}}{e \gamma}=c_{0}, & \operatorname{CS}\left(X_{j}, X_{k}\right)=\frac{c_{0}^{2}}{e}=c_{0} .
\end{array}
$$

Thus the pairs $\left(X_{i}, X_{j}\right)$ and $\left(X_{i}, X_{k}\right)$ are exotic quasilinear, and so $X_{j}, X_{k} \subset \mathrm{QL}\left(X_{i}\right)$, while $Y_{i}$ is g-anisotropic (i.e., not g-isotropic). Thus $Y_{i} \in \mathrm{QL}\left(X_{i}\right)$, but $Y_{i} \notin\left[X_{j}, X_{k}\right]$. We have the following picture with three non-convex $Q L$-stars $\mathrm{QL}\left(X_{1}\right), \mathrm{QL}\left(X_{2}\right)$, and $\mathrm{QL}\left(X_{3}\right)$.

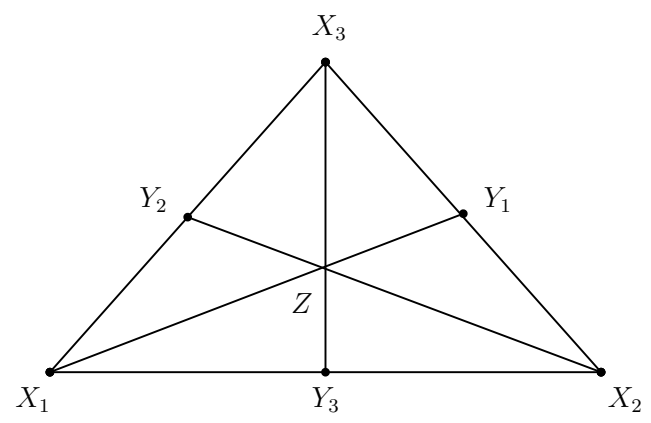

The three ray intervals $\left[X_{1}, Y_{1}\right],\left[X_{2}, Y_{2}\right]$, and $\left[X_{3}, Y_{3}\right]$ meet at $Z=\operatorname{ray}\left(\varepsilon_{1}+\varepsilon_{2}+\varepsilon_{3}\right)$.

Theorem 7.4. Assume that $X_{1}, X_{2}, X_{3}$ are rays in $V$ where $X_{1}$ is g-isotropic, $X_{3}$ is $g$ anisotropic, and

$$
\operatorname{CS}\left(X_{1}, X_{2}\right) \leq \operatorname{CS}\left(X_{1}, X_{3}\right)=c_{0} .
$$

Then $\mathrm{QL}\left(X_{1}\right)$ is not convex, namely there exist $g$-isotropic rays $Y_{1}, Y_{2}$ and a $g$-anisotropic ray $Z$ with $Z \in\left[Y_{1}, Y_{2}\right] \subset\left[X_{1}, X_{2}\left[\right.\right.$ and $\operatorname{CS}\left(X_{1}, Y_{1}\right)=\operatorname{CS}\left(X_{1}, Y_{2}\right)=\operatorname{CS}\left(X_{1}, Z\right)=c_{0}$, whence $Y_{1}, Y_{2} \in \mathrm{QL}\left(X_{1}\right), Z \notin \mathrm{QL}\left(X_{1}\right)$.

Proof. We choose vectors $\varepsilon_{i} \in V$ for which $X_{i}=\operatorname{ray}\left(\varepsilon_{i}\right)$,

$$
\begin{aligned}
& \alpha_{1}:=q\left(\varepsilon_{1}\right) \in \mathcal{G}, \quad \alpha_{2}:=q\left(\varepsilon_{2}\right) \in R \backslash\{0\}, \\
& \alpha_{3}:=q\left(\varepsilon_{3}\right) \in \mathcal{T}, \quad \alpha_{23}:=b\left(\varepsilon_{2}, \varepsilon_{3}\right) \in R .
\end{aligned}
$$

The rays $X$ in $\left[X_{2}, X_{3}\right.$ [ have a presentation $X=\operatorname{ray}\left(\varepsilon_{2}+\lambda \varepsilon_{3}\right)$ with $\lambda$ running through $R$. If $e \lambda$ is big enough, then

$$
\begin{array}{ll}
q\left(\varepsilon_{2}+\lambda \varepsilon_{3}\right) & =\alpha_{2}+\lambda \alpha_{23}+\lambda^{2} \alpha_{3}=\lambda^{2} \alpha_{3}, \\
\operatorname{CS}\left(\varepsilon_{1}, \varepsilon_{2}+\lambda \varepsilon_{3}\right) & =\frac{\alpha_{12}^{2}+\lambda^{2} \alpha_{13}^{2}}{\alpha_{1} \lambda^{2} \alpha_{3}}=\frac{\alpha_{12}^{2}}{\lambda^{2} \alpha_{1} \alpha_{2}} \frac{\alpha_{2}}{\alpha_{3}}+\frac{\alpha_{13}^{2}}{\alpha_{1} \alpha_{3}} \\
& =\operatorname{CS}\left(\varepsilon_{1}, \varepsilon_{3}\right)=c_{0} .
\end{array}
$$

More precisely, this holds if $\lambda \geq_{\nu} \lambda_{0}$ for some $\lambda_{0}$ with

$$
\lambda_{0}^{2} \geq_{\nu} \max \left(\frac{\alpha_{2}}{\alpha_{3}}, \frac{\alpha_{3}^{2}}{\alpha_{23}^{2}}\right) .
$$

Now choose scalars $\lambda_{1}>_{\nu} \rho>_{\nu} \lambda_{2} \geq_{\nu} \lambda_{0}$ where $\lambda_{1}, \lambda_{2} \in \mathcal{G}, \rho \in \mathcal{T}$, and take $Y_{i}=\operatorname{ray}\left(\varepsilon_{2}+\right.$ $\left.\lambda_{i} \varepsilon_{3}\right), i=1,2, Z=\operatorname{ray}\left(\varepsilon_{2}+\rho \varepsilon_{3}\right)$. 


\section{ENLARGEMENTS OF QL-PATHS, AND BRIDGES TO FIND SHORT QL-PATHS}

In this section we only assume, that the pair $(R, V)$ is ray-admissible (cf. (4.1), (4.2)), and that $(q, b)$ is a quadratic pair on $V$ with $q$ anisotropic.

Definition 8.1. A $\boldsymbol{Q L}$-path in $\operatorname{Ray}(V)$ is a sequence $\left(X_{0}, X_{1}, \ldots, X_{n}\right)$ of rays in $V$ such that every pair $\left(X_{i}, X_{i+1}\right), 0 \leq i<n$, is quasilinear (equivalently, that the closed interval $\left[X_{i}, X_{i+1}\right]$ is quasilinear $)$. We say that the $Q L$-path $\left(X_{0}, \ldots, X_{n}\right)$ has length $n$ and runs from $X_{0}$ to $X_{n}$.

This definition has a graph theoretic flavor.

Definition 8.2. We define a (simple, undirected) graph $\Gamma_{\mathrm{QL}}(V, q)$ to be the graph whose vertices are the rays in $V$, and its edges are the quasilinear pairs $(X, Y)$ of rays. For formal reasons we admit loops in $\Gamma_{\mathrm{QL}}(V, q)$. For every $X \in \operatorname{Ray}(V)$ we have a loop $(X, X)$ due to the fact that $\operatorname{CS}(X, X) \leq e$, cf. [5, Eq. (1.9)] 6 We call $\Gamma_{\mathrm{QL}}(V, q)$ the quasilinear graph of $(V, q)$.

Note that this graph does not depend on the choice of the companion $b$ of $q$, since the sets $\mathrm{QL}(X)$ are independent of the choice of $b$.

We define "enlargements" of a given QL-path $\left(X_{0}, \ldots, X_{n}\right)$ and use them to develop procedures for replacing $\left(X_{0}, \ldots, X_{n}\right)$ by a path of shorter length from $X_{0}$ to $X_{n}$ under suitable conditions.

Notation 8.3. We refine the graph $\Gamma_{\mathrm{QL}}(V, q)$ by replacing an edge $X-Y$ by an arrow $X \rightarrow Y$ in the case that $X \preceq_{\mathrm{QL}} Y$, i.e., $\mathrm{QL}(X) \subset \mathrm{QL}(Y)$, and consequently replace $X-Y$ by an arrow with two heads $X \leftrightarrow Y$, if $X \sim_{\mathrm{QL}} Y$, i.e., $\mathrm{QL}(X)=\mathrm{QL}(Y)$. But most often we then abusively identify $X=Y$, since in all matters below a vertex $X$ can be replaced by a $Q L$-equivalent ray. We call the new diagram the decorated quasilinear graph $\widetilde{\Gamma}_{\mathrm{QL}}(V, q)$ of $(V, q)$.

Definition 8.4. Given two QL-paths $\left(X_{0}, X_{1}, \ldots, X_{n}\right)$ and $\left(Y_{0}, Y_{1}, \ldots, Y_{n}\right)$ of same length $n$, we say that $\left(Y_{0}, Y_{1}, \ldots, Y_{n}\right)$ is an enlargement of $\left(X_{0}, X_{1}, \ldots, X_{n}\right)$, if $\mathrm{QL}\left(Y_{i}\right) \supset \mathrm{QL}\left(X_{i}\right)$ for $0 \leq i \leq n$. Then we have the following subdiagram of $\widetilde{\Gamma}_{\mathrm{QL}}(V, q)$ :

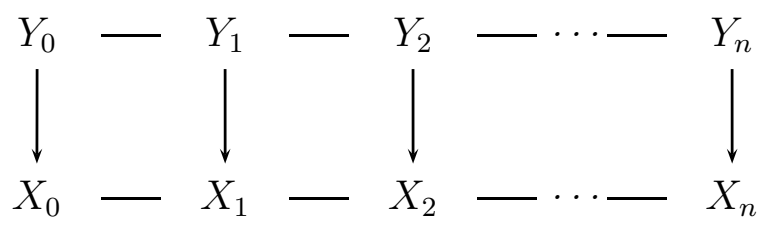

Thus, by using the mother set $\left\{Y_{i}, Y_{i+1}\right\}$, we have enlarged the quasilinear interval $\left[X_{i}, X_{i+1}\right]$ to the convex hull of $\left\{X_{i}, X_{i+1}, Y_{i}, Y_{i+1}\right\}$. Note that $X_{i}=Y_{i}$, if the associated disjoint mother set is $\left\{Y_{i+1}\right\}$, and that $X_{i+1}=Y_{i+1}$ if this set is $\left\{Y_{i}\right\}$.

\footnotetext{
${ }^{6}$ If $(q, b)$ is balanced, then $\operatorname{CS}(X, X)=e$ for every $X$ [5, Eq. (1.10)].
} 
In the diagram (8.1) we can always enrich a square

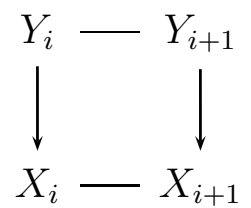

to the subdiagram

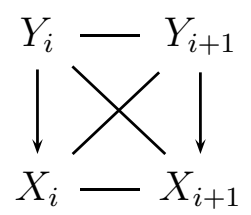

of $\widetilde{\Gamma}_{\mathrm{QL}}(V, q)$.

In other words, $X_{i} \in \mathrm{QL}\left(Y_{i+1}\right)$ and $X_{i+1} \in \mathrm{QL}\left(Y_{i}\right)$. Moreover, it may happen, say, if $0<i<n$, that there are indices $j<i-1$ and $k>i+1$ with $X_{j} \in \mathrm{QL}\left(Y_{i}\right), X_{k} \in \mathrm{QL}\left(Y_{i}\right)$. Then we have the following subdiagram of $\widetilde{\Gamma}_{\mathrm{QL}}(V, q)$ with $Y:=Y_{i}$

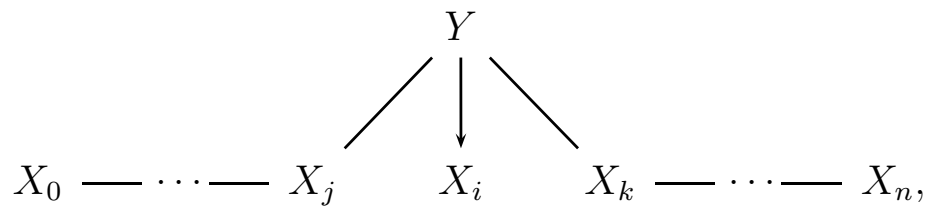

which gives a QL-path $\left(X_{0}, \ldots, X_{j}, Y, X_{k}, \ldots X_{n}\right)$ from $X_{0}$ to $X_{n}$ of shorter length $j+1+n-$ $k=n-(k-j-1)$. Also there may exist two different rays $Y^{\prime}, Y^{\prime \prime}$ with $\mathrm{QL}\left(Y^{\prime}\right) \supset \mathrm{QL}\left(X_{i}\right)$, $\mathrm{QL}\left(Y^{\prime \prime}\right) \supset \mathrm{QL}\left(X_{i}\right)$, so that we have an index $r<j$ and an index $s>k$ with $X_{r} \in \mathrm{QL}\left(Y^{\prime}\right)$, $X_{s} \in \mathrm{QL}\left(Y^{\prime \prime}\right)$. Then we have a subdiagram

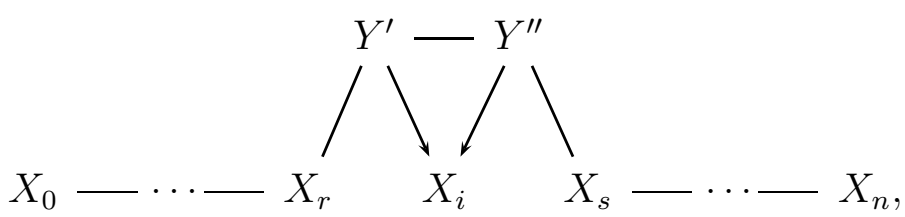

of $\widetilde{\Gamma}_{\mathrm{QL}}(q)$ which gives a path from $X_{0}$ to $X_{n}$ of even smaller length $r+2+(n-s)=$ $n-(s-r-2) .<n-(k-j-1)$. In more imaginative terms, we have built "bridges" in (8.3) and (8.4) to span the subpaths $\left(X_{j}, X_{j+1}, \ldots, X_{k}\right)$ and $\left(X_{r}, \ldots, X_{s}\right)$ respectively by use of one or two rays in $\operatorname{sat}_{\mathrm{QL}}\left(\mathrm{X}_{\mathrm{i}}\right)$ as "pillars".

We are ready for a formal definition of a bridge. Assume that $\left(X_{0}, X_{1}, \ldots, X_{n}\right)$ is any QL-path in $\operatorname{Ray}(V)$.

Definition 8.5. A bridge over $\left(X_{0}, X_{1}, \ldots, X_{n}\right)$ (or spanning $\left(X_{0}, X_{1}, \ldots, X_{n}\right)$ ) is a $Q L$ path $\left(X_{0}, Y_{1}, \ldots, Y_{m}, X_{n}\right)$ together with a sequence $0 \leq c(1)<c(2)<\cdots<c(m) \leq n$ such that

$$
Y_{r} \in \operatorname{sat}_{\mathrm{QL}}\left(\mathrm{X}_{\mathrm{c}(\mathrm{r})}\right)
$$

for $1 \leq r \leq m$, and furthermore $c(2) \geq 2$ in the case $c(1)=0$, and $c(m-1) \leq n-2$, in the case $c(m)=n$.

Note that we do not exclude the possibility that $Y_{r}=X_{c(r)}$ for some indices $r$. 
Comment 8.6. In the case $0<c(1), c(m)<n$, a bridge over $\left(X_{0}, X_{1}, \ldots, X_{n}\right)$ is given by a diagram

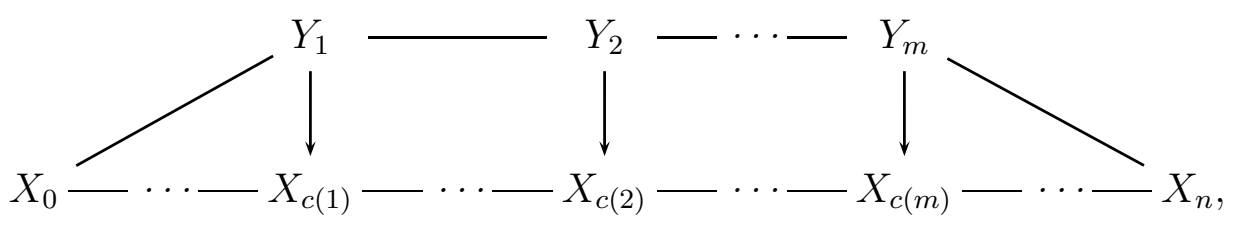

in $\widetilde{\Gamma}_{\mathrm{QL}}(V, q)$, while, if say $c(1)=0, c(m)<n$, we have a diagram

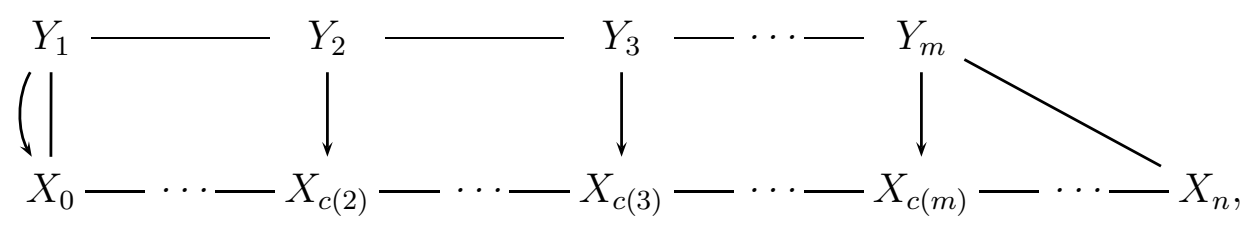

If we would allow here $c(2)=1$, we could omit the ray $Y_{1}$ in the $Q L$-path $\left(X_{0}, Y_{1}, \ldots, Y_{m}, X_{n}\right)$ (cf. (8.2)) and would obtain for free the shorter bridge

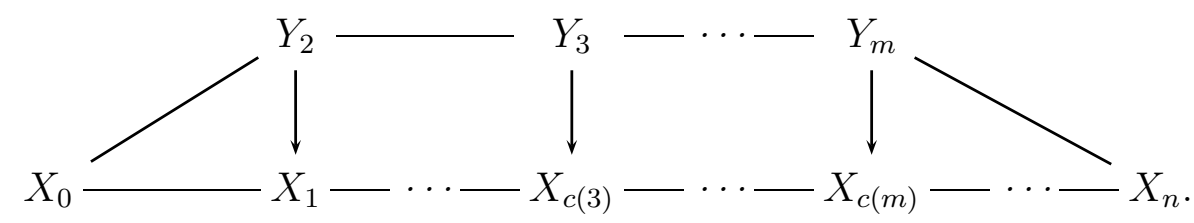

We want to discard this annoying triviality.

Comment 8.7. Our formal definition of bridges does not include the "bridge" (8.4) with a doubled pillar. But it includes an equivalent object. Assume that $X_{i-1}=X_{i}$ in a given path $\left(X_{0}, X_{1}, \ldots, X_{n}\right)$, i.e., the QL-path $\left(X_{0}, \ldots, X_{n}\right)$ which contains the loop $\left(X_{i}, X_{i}\right)$ of $\Gamma_{\mathrm{QL}}(V, q)$. Then the interval $\left[X_{i-1}, X_{i}\right]$ shrinks to the one-point set $\left\{X_{i}\right\}$. The diagram (8.4) shows in essence the same objects as the bridge

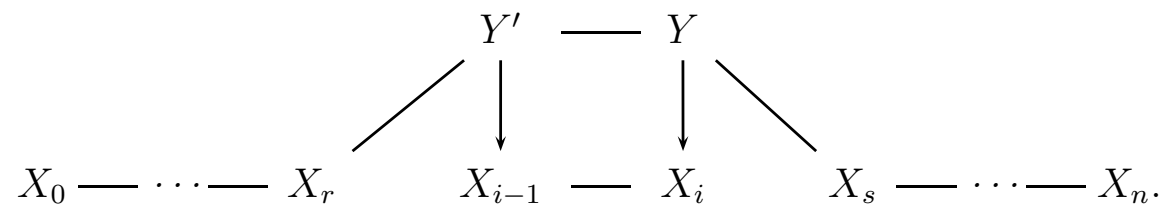

We note an important fact, immediately obtained from the definition 14.5 of bridges.

Proposition 8.8. Assume that $\left(X_{0}, Y_{1}, \ldots, Y_{m}, X_{n}\right)$ is a bridge over $\left(X_{0}, X_{1}, \ldots, X_{n}\right)$. Then any bridge $\left(X_{0}, Z_{1}, \ldots, Z_{q}, X_{n}\right)$ spanning the $Q L$-path $\left(X_{0}, Y_{1}, \ldots, Y_{m}, X_{n}\right)$ is again a bridge over $\left(X_{0}, X_{1}, \ldots, X_{n}\right)$.

We describe a procedure to shorten a given QL-path $\left(X_{0}, X_{1}, \ldots, X_{n}\right)$ without yet using enlargements of $\left(X_{0}, X_{1}, \ldots, X_{n}\right)$ ]

Definition 8.9. A basic reduction of the $Q L$-path $\left(X_{0}, \ldots, X_{n}\right)$ arises as follows. Pick some $i \in[0, n]$.

\footnotetext{
${ }^{7}$ An analogous procedure can be performed in any simple graph.
} 
(a) If there exist indices $k>i+1$ such that the pair $\left(X_{i}, X_{k}\right)$ is quasilinear, let s denote the maximal one of these and replace $\left(X_{0}, \ldots, X_{n}\right)$ by $\left(X_{0}, \ldots, X_{i}, X_{s}, \ldots, X_{n}\right)$ omitting all rays $X_{p}$ with $i<p<s$.

(b) If there exist indices $j<i-1$ such that $\left(X_{j}, X_{i}\right)$ is quasilinear, let $r$ denote the minimal of these, and replace $\left(X_{0}, \ldots, X_{n}\right)$ by $\left(X_{0}, \ldots, X_{r}, X_{i}, \ldots, X_{n}\right)$, omitting all rays $X_{p}$ with $r<p<i$.

More precisely we call a $Q L$-path $\left(X_{0}, \ldots, X_{i}, X_{s}, \ldots, X_{n}\right)$ as in (a) an $f$-basic reduction (= forward basic reduction) of $\left(X_{0}, \ldots, X_{n}\right)$ and a $Q L$-path $\left(X_{0}, \ldots, X_{r}, X_{i}, \ldots, X_{n}\right)$ as in (b) a b-basic reduction (= backward basic reduction) of $\left(X_{0}, \ldots, X_{n}\right)$.

Definition 8.10. We say that a $Q L$-path $\left(X_{0}, X_{1}, \ldots, X_{n}\right)$ is direct, if $X_{0} \neq X_{n}$ and there do not exist indices $i, j \in[0, n]$ with $|i-j| \geq 2$, such that the pair $\left(X_{i}, X_{j}\right)$ is quasilinear. (Note that this implies $X_{i} \neq X_{i+1}$ for $0 \leq i<n$.)

The following is obvious from Definitions 8.9 and 8.10 .

\section{Proposition 8.11.}

(a) A QL-path $\left(X_{0}, \ldots, X_{n}\right)$ with $X_{0} \neq X_{n}$ is direct iff no forward basic reduction of $\left(X_{0}, \ldots, X_{n}\right)$ exists, iff no backward basic reduction of $\left(X_{0}, \ldots, X_{n}\right)$ exists.

(b) Any QL-path $\left(X_{0}, \ldots, X_{n}\right)$ with $X_{0} \neq X_{n}$ can be reduced to a direct QL-path by finitely many (at most $n-1$ ) such reductions.

Remark 8.12. It may happen that $\left(X_{0}, Y_{1}, \ldots, X_{n}\right)$ can be reduced in this way to different direct QL-paths. Assume for example that $n=6$ and that $\left(X_{0}, X_{2}\right)$ and $\left(X_{1}, X_{5}\right)$ are the only quasilinear pairs $\left(X_{i}, X_{j}\right)$ with $0 \leq i, j \leq n$ and $j-i \geq 2$. Then omitting the ray $X_{1}$ in $\left(X_{0}, \ldots, X_{6}\right)$ gives us a direct path of length 5 , while omitting $X_{2}, X_{3}, X_{4}$ gives us a direct path of length 3.

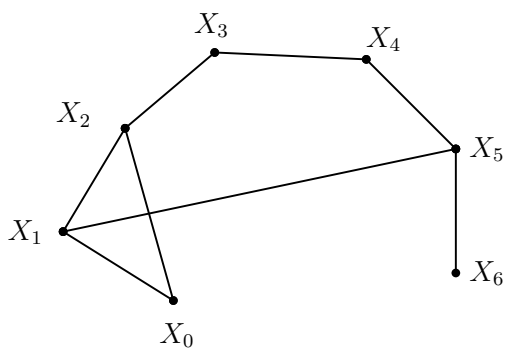

We now describe a procedure to shorten a QL-path by use of enlargements.

Procedure 8.13. Given a QL-path $\left(X_{0}, \ldots, X_{n}\right)$ with $X_{0} \neq X_{n}$, we pick an index $i \in[0, n]$ and choose a ray $Y$ in $\operatorname{sat}_{\mathrm{QL}}\left(\mathrm{X}_{\mathrm{i}}\right)$, i.e., with $\mathrm{QL}(Y) \supset \mathrm{QL}\left(X_{i}\right)$.

a) If $i>0$ and $X_{k} \in \mathrm{QL}(Y)$ for some $k>i+1$, let $s$ denote the maximal index $\leq n$ with $X_{s} \in \mathrm{QL}(Y)$ and build the bridge

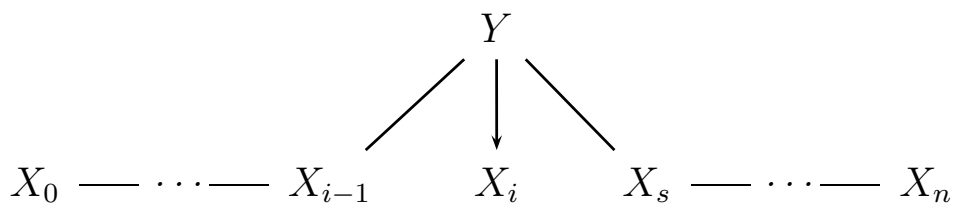


over $\left(X_{0}, \ldots, X_{n}\right)$. This gives us a path $\left(X_{0}, \ldots, X_{i-1}, Y, X_{s}, \ldots, X_{n}\right)$ with $s>i+1$ of length $n-(s-i-1)$. If $i=0$, do the same, provided there is an index $k>2$ with $X_{k} \in \mathrm{QL}(Y)$. This gives us a bridge

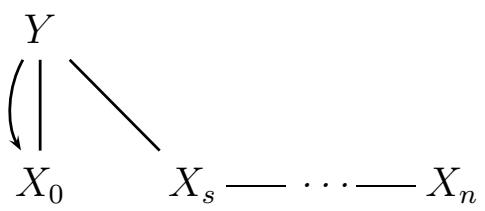

and a path $\left(X_{0}, Y, X_{s}, \ldots, X_{n}\right)$ with $s>2$ of length $n-(s-2)$.

b) If $i<n$ and there exists an index $j<i-1$ with $X_{j} \in \mathrm{QL}(Y)$, let $r$ denote the minimal index with $X_{r} \in \mathrm{QL}(Y)$ and build the bridge

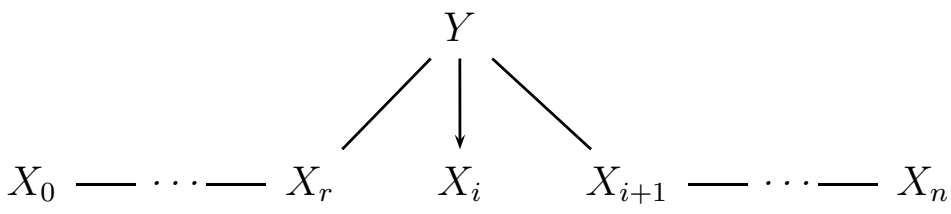

over $\left(X_{0}, \ldots, X_{n}\right)$. This gives us a path $\left(X_{0}, \ldots, X_{r}, Y, X_{i+1}, \ldots, X_{n}\right)$ with $r<i-1$ of length $n-(i-1-r)$. If $i=n$, do the same, provided there exists an index $j<n-2$ with $X_{j} \in \mathrm{QL}(Y)$. This gives us the bridge

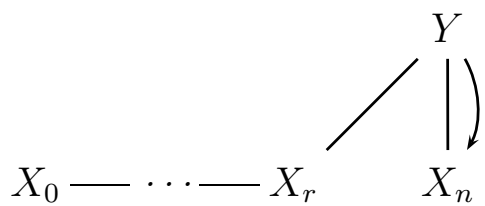

and the path $\left(X_{0}, \ldots, X_{r}, Y, X_{n}\right)$ with $r<n-2$ of length $r+2<n$.

Definition 8.14. We call the paths so obtained elementary reductions of $\left(X_{0}, \ldots, X_{n}\right)$, more precisely, those obtained in a) f-elementary (= forward elementary) reductions, and those obtained in b) b-elementary (= backward elementary) reductions of $\left(X_{0}, \ldots, X_{n}\right)$. We further call the bridges (8.9) -(8.12) elementary bridges over $\left(X_{0}, \ldots, X_{n}\right)$ (or spanning $\left.\left(X_{0}, \ldots, X_{n}\right)\right)$.

Definition 8.15. We call a QL-path $\left(X_{0}, X_{1}, \ldots, X_{n}\right)$ optimal, if $n \geq 3, X_{0} \neq X_{n}$, and the path does not admit any elementary reduction.

Observe that this implies $X_{0} \neq X_{n}$, since in the case $X_{0}=X_{n}$ we would have a bridge (8.10) with $Y=X_{0}$ and $s=n$. Since an elementary reduction of a QL-path has shorter length than the given one, it is plain that any QL-path $\left(X_{0}, \ldots, X_{n}\right)$ with $n \geq 3, X_{0} \neq X_{n}$ becomes either optimal or direct of length $\leq 2$.

Proposition 8.16. Every optimal QL-path is a direct QL-path.

Proof. Let $\left(X_{0}, \ldots, X_{n}\right)$ be a QL-path of length $n \geq 3$ which is not direct. We verify that $\left(X_{0}, \ldots, X_{n}\right)$ admits an elementary reduction, and then will be done. There are indices $i, j \in[0, n]$ with $j \geq i+2$ and $\left(X_{i}, X_{j}\right)$ quasilinear. Fixing $i$, let $s$ denote the maximal such index $j$. If $i>0$, or if $i=0, s \geq 3$, we have an $f$-elementary reduction obtained by a 
bridge (8.9) or (8.10) with $Y=X_{i}$. There remains the case $i=0, s=2$. Now $\left(X_{0}, X_{2}\right)$ is quasilinear, and so we have a $b$-elementary reduction $\left(X_{0}, X_{2}, \ldots, X_{n}\right)$ by the bridge (8.11), there with $i=2, r=0, Y=X_{2}$.

For any ray $X$ in $V$ let $\widehat{Q}(X)$ denote the union of all QL-stars containing $\mathrm{QL}(X)$. In other terms,

$$
\widehat{\mathrm{QL}}(X):=\bigcup\left\{\mathrm{QL}(Y) \mid Y \in \operatorname{sat}_{\mathrm{QL}}(\mathrm{X})\right\} \text {. }
$$

It is obvious from the definition of elementary reductions and optimal paths (Definitions 8.14 and 8.15) that the following holds.

Scholium 8.17. A QL-path $\left(X_{0}, \ldots, X_{n}\right)$ with $n \geq 3, X_{0} \neq X_{n}$ is optimal iff

$$
\widehat{\mathrm{QL}}\left(X_{i}\right) \cap\left\{X_{0}, \ldots, X_{n}\right\}=\left\{X_{i-1}, X_{i}, X_{i-1}\right\},
$$

for $0<i<n$, while

$$
\widehat{\mathrm{QL}}\left(X_{0}\right) \cap\left\{X_{0}, \ldots, X_{n}\right\} \subset\left\{X_{0}, X_{1}\right\}
$$

and

$$
\widehat{\mathrm{QL}}\left(X_{n}\right) \cap\left\{X_{0}, \ldots, X_{n}\right\} \subset\left\{X_{n-1}, X_{n}\right\}
$$

We look for a characterization of optimal paths by properties of their enlargements.

Theorem 8.18. Assume that $\left(X_{0}, \ldots, X_{n}\right)$ is a $Q L$-path with $X_{0} \neq X_{n}$. The following are equivalent.

(i) $\left(X_{0}, \ldots, X_{n}\right)$ is optimal.

(ii) For every $i \in[0, n]$ and $Y \in \operatorname{sat}_{\mathrm{QL}}\left(\mathrm{X}_{\mathrm{i}}\right)$ is $\left(X_{0}, \ldots, X_{i-1}, Y, X_{i+1}, \ldots, X_{n}\right)$ a direct QL-path.

(iii) For every $i \in[0, n]$ and $Y \in \operatorname{sat}_{\mathrm{QL}}\left(\mathrm{X}_{\mathrm{i}}\right)$ there exists a direct enlargement $\left(Z_{0}, \ldots, Z_{n}\right)$ (i.e., an enlargement which is a direct $Q L$-path) of $\left(X_{0}, \ldots, X_{n}\right)$ with $\mathrm{QL}\left(Z_{i}\right) \supset$ $\mathrm{QL}(Y)$.

Proof. (i) $\Leftrightarrow$ (ii): Evident from Scholium 8.17 ,

(ii) $\Rightarrow$ (iii): Trivial, since $\left(X_{0}, \ldots, X_{i-1}, Y, X_{i-1}, \ldots, X_{n}\right)$ is an enlargement of $\left(X_{0}, \ldots, X_{n}\right)$.

(iii) $\Rightarrow$ (ii): Let $Y \in \operatorname{sat}_{\mathrm{QL}}\left(\mathrm{X}_{\mathrm{i}}\right)$. Suppose there exists $j \in[0, n]$ with $|j-i|>1$ and $X_{j} \in$ $\mathrm{QL}(Y)$. Choose a direct enlargement $\left(Z_{0}, \ldots, Z_{n}\right)$ of $\left(X_{0}, \ldots, X_{n}\right)$ with $\mathrm{QL}\left(Z_{i}\right) \supset \mathrm{QL}(Y)$. Then $X_{j} \in \mathrm{QL}\left(Z_{i}\right)$, and so $Z_{i} \in \mathrm{QL}\left(X_{j}\right)$. Since $\mathrm{QL}\left(X_{j}\right) \subset \mathrm{QL}\left(Z_{j}\right)$, this implies $Z_{i} \in \mathrm{QL}\left(Z_{j}\right)$, contradicting our hypothesis that $\left(Z_{0}, \ldots, Z_{n}\right)$ is direct.

Theorem 8.18 can be stated in a more conceptual way by use of a quasiordering $\preceq$ QL on the set of all QL-paths of fixed length.

Definition 8.19. For any two $Q L$-paths $\left(X_{0}, \ldots, X_{n}\right),\left(Y_{0}, \ldots, Y_{n}\right)$, we say that $\left(Y_{0}, \ldots, Y_{n}\right)$ dominates $\left(X_{0}, \ldots, X_{n}\right)$, and write $\left(X_{0}, \ldots, X_{n}\right) \preceq_{\mathrm{QL}}\left(Y_{0}, \ldots, Y_{n}\right)$, if $X_{i} \preceq_{\mathrm{QL}} Y_{i}$ for all $0 \leq i \leq n$, in other terms, $\mathrm{QL}\left(X_{i}\right) \subset \mathrm{QL}\left(Y_{i}\right)$ for every $0 \leq i \leq n$.

Theorem 8.20. A QL-path $\left(X_{0}, \ldots, X_{n}\right)$ is optimal iff the set of all direct enlargements of $\left(X_{0}, \ldots, X_{n}\right)$ is cofinal in the set of all enlargements of $\left(X_{0}, \ldots, X_{n}\right)$, i.e., every enlargement $\left(Y_{0}, \ldots, Y_{n}\right)$ of $\left(X_{0}, \ldots, X_{n}\right)$ is dominated by some enlargement $\left(Z_{0}, \ldots, Z_{n}\right)$ of $\left(X_{0}, \ldots, X_{n}\right)$ which is a direct path. 
Proof. We verify the equivalence of this condition with condition (iii) in Theorem 8.18 . It is plain that the new condition implies condition (iii). On the other hand, if an enlargement $\left(Y_{0}, \ldots, Y_{n}\right)$ of $\left(X_{0}, \ldots, X_{n}\right)$ is given and (iii) holds, we find for every $i \in[0, n]$ a ray $Z_{i}$ with $\mathrm{QL}\left(Z_{i}\right) \supset \mathrm{QL}\left(Y_{i}\right)$ and $X_{j} \notin \mathrm{QL}\left(Z_{i}\right)$ for $|i-j|>1$. Now $\left(Z_{0}, \ldots, Z_{n}\right)$ is a direct enlargement of $\left(X_{0}, \ldots, X_{n}\right)$ dominating $\left(Y_{0}, \ldots, Y_{n}\right)$ (cf. Scholium 8.17).

We add an observation which enriches the picture around this theorem.

Proposition 8.21. Assume that $\left(X_{0}, \ldots, X_{n}\right)$ is a QL-path which is dominated by a direct QL-path $\left(Y_{0}, \ldots, Y_{n}\right)$. Then $\left(X_{0}, \ldots, X_{n}\right)$ itself is direct.

Proof. Assume that $i<j$ are indices in $[0, n]$ such that the pair $\left(X_{i}, X_{j}\right)$ is quasilinear, in other terms, $X_{j} \in \mathrm{QL}\left(X_{i}\right)$. We have $\mathrm{QL}\left(X_{i}\right) \subset \mathrm{QL}\left(Y_{i}\right)$ and $\mathrm{QL}\left(X_{j}\right) \subset \mathrm{QL}\left(Y_{j}\right)$, and so $X_{j} \in \mathrm{QL}\left(Y_{i}\right)$, whence $Y_{i} \in \mathrm{QL}\left(X_{j}\right)$ and then $Y_{i} \in \mathrm{QL}\left(Y_{j}\right)$. Since $\left(Y_{0}, \ldots Y_{n}\right)$ is direct, it follows that $j=i+1$, as desired.

It can happen that every enlargement of an optimal path $\left(X_{0}, \ldots, X_{n}\right), n \geq 3$, is again optimal, cf. \$12 below.

\section{INTERPLAY OF THE QUASILINEAR ORDERING ON THE RAY SPACE WITH DIRECT QL-PATHS}

In this section we study an interplay of the (partial) quasiordering $\preceq_{\mathrm{QL}}$ on $\operatorname{Ray}(V)$ with the direct QL-paths (in particular the optimal QL-paths) in $V$. As in $\oint 8$ we only assume that $(R, V)$ is ray-admissible and $(q, b)$ is a quadratic pair on $V$ with $q$ anisotropic.

Given a ray $X$ in $V$, we denote the upset and downset of $X$ w.r. to $\preceq_{\mathrm{QL}}$ by $X^{\uparrow}$ and $X^{\downarrow}$, i.e.,

$$
X^{\uparrow}=\{Z \in \operatorname{Ray}(V) \mid \mathrm{QL}(X) \subset \mathrm{QL}(Z)\}=\operatorname{sat}_{\mathrm{QL}}(\mathrm{X})
$$

in previous terminoloy, and

$$
X^{\downarrow}=\{Z \in \operatorname{Ray}(V) \mid \mathrm{QL}(Z) \subset \mathrm{QL}(X)\} .
$$

More generally we denote for any set $S$ in $\operatorname{Ray}(V)$ the up- and downsets of $S$ by $S^{\uparrow}$ and $S^{\downarrow}$, i.e.,

$$
S^{\uparrow}=\bigcup_{X \in S} X^{\uparrow}, \quad S^{\downarrow}=\bigcup_{X \in S} X^{\downarrow}
$$

Theorem 9.1. Assume that $\left(Z_{0}, \ldots, Z_{n}\right)$ is an enlargement of an optimal QL-path $\left(X_{0}, \ldots, X_{n}\right)$ in $V$. Then the sets

$$
\left\{Z_{0}, Z_{1}\right\}^{\uparrow}, Z_{2}^{\uparrow}, \ldots, Z_{n-1}^{\uparrow},\left\{Z_{n-1}, Z_{n}\right\}^{\uparrow}
$$

are mutually disjoint, and so form a partition of the set $\left\{Z_{0}, \ldots, Z_{n}\right\}^{\uparrow}$.

Proof. Since $Z_{i}^{\uparrow} \subset X_{i}^{\uparrow}$, it suffices to verify this for the optimal QL-path $\left(X_{0}, \ldots, X_{n}\right)$ itself. Suppose that there exists a ray $Y$ with $Y \in X_{p}^{\uparrow} \cap X_{q}^{\uparrow}$ for different indices $p, q$ in $[0, n]$. If $0<p<q<n$ we would obtain a diagram 


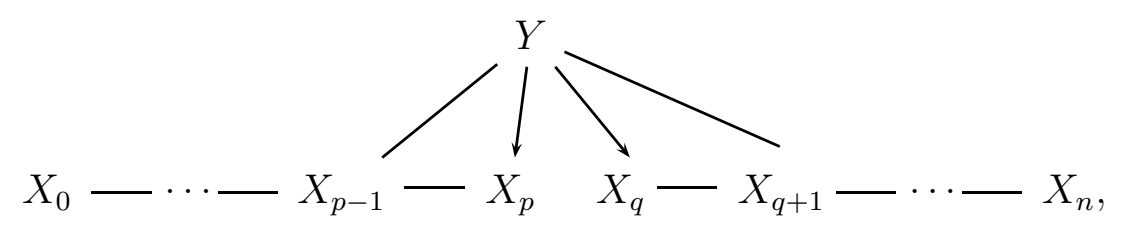

and so a QL-path $\left(X_{0}, \ldots, X_{p-1}, Y, X_{q+1}, \ldots, X_{n}\right)$ which is both an $f$-elementary and a $b$ elementary reduction of $\left(X_{0}, \ldots, X_{n}\right)$ in contradiction to our assumption that $\left(X_{0}, \ldots, X_{n}\right)$ is optimal. If $p=0, q \geq 2$ we would obtain a diagram

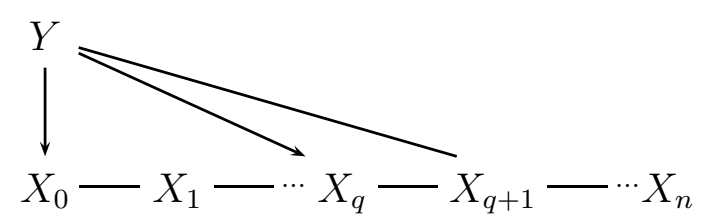

and a QL-path $\left(X_{0}, Y, X_{q+1}, \ldots, X_{n}\right)$ which is an $f$-elementary reduction of $\left(X_{0}, \ldots, X_{n}\right)$ in contradiction to our assumption that $\left(X_{0}, \ldots, X_{n}\right)$ is optimal. In the same way we see that $p \leq n-2, q=n$ is impossible. Thus any two of the sets listed in the theorem have empty intersection.

Corollary 9.2. Assume that $\left(Z_{0}, \ldots, Z_{n}\right)$ is an enlargement of an optimal QL-path. Then the downset $Y^{\downarrow}$ of any ray $Y$ in $V$ meets each of the three sets $\left\{Z_{1}, \ldots, Z_{n-1}\right\},\left\{Z_{0}, Z_{2}, \ldots, Z_{n}\right\}$, $\left\{Z_{0}, \ldots, Z_{n-2}, Z_{n}\right\}$ in at most one ray.

Proof. If $Y^{\downarrow}$ contains two rays $Z_{i}, Z_{j}(0 \leq i<j \leq n)$, then $Y \in Z_{i}^{\uparrow} \cap Z_{j}^{\uparrow}$. This is excluded by Theorem 9.1 , if $Z_{i}$ and $Z_{j}$ are elements of one of these three sets.

The set of enlargements of a given QL-path $\left(X_{0}, \ldots, X_{n}\right)$ can be very rich, as is indicated by the following fact.

Proposition 9.3. Assume that $\left(Y_{0}, \ldots, Y_{n}\right)$ is an enlargement of $\left(X_{0}, \ldots, X_{n}\right)$. Then every sequence of rays $\left(Z_{0}, \ldots, Z_{n}\right)$ with $Z_{i} \in\left[X_{i}, Y_{i}\right]$ for $0 \leq i \leq n$ is again an enlargement of $\left(X_{0}, \ldots, X_{n}\right)$ (but often $\left(Y_{0}, \ldots, Y_{n}\right)$ is not an enlargement of $\left(Z_{0}, \ldots, Z_{n}\right)$ ).

Proof. $Z_{i} \in X_{i}^{\uparrow}$, since by Theorem 5.6 each set $X_{i}^{\uparrow}$ is convex. Furthermore, each pair $\left(Z_{i}, Z_{i+1}\right), 0 \leq i<n$, is quasilinear, since the convex hull of $\left\{X_{i}, X_{i+1}, Y_{i}, Y_{i+1}\right\}$ is convex, as we know for long.

Theorem 9.4. Assume that $\left(X_{0}, \ldots, X_{n}\right)$ is a direct $Q L$-path and $Y$ is a ray in $V$ with $Y^{\uparrow} \cap\left\{X_{0}, \ldots, X_{n}\right\} \neq \emptyset$. Then $Y^{\uparrow}$ meets the set $\left\{X_{0}, \ldots, X_{n}\right\}$ either in exactly one ray $X_{p}$ or in exactly two rays $X_{p}, X_{p+1}$. In the first case $X_{i} \notin \mathrm{QL}(Y)$ for $|i-p|>1$ if $0<p<n$, while $X_{i} \notin \mathrm{QL}(Y)$ for $i \geq 2$ if $p=0$, and $X_{i} \notin \mathrm{QL}(Y)$ for $i \leq n-2$ if $p=n$. In the second case $X_{i} \notin \mathrm{QL}(Y)$ for $i \notin\{p, p+1\}$.

Proof. All assertions are immediate consequences of the following three observations.

a) Assume that $X_{p} \in Y^{\uparrow}$ for some $p \in[0, n]$ and that $X_{i} \in \mathrm{QL}(Y)$ for some $i \in[0, n]$. Then $X_{i} \in \mathrm{QL}\left(X_{p}\right)$ because $\mathrm{QL}(Y) \subset \mathrm{QL}\left(X_{p}\right)$. Since the path $\left(X_{0}, \ldots, X_{n}\right)$ is direct, this implies $|i-p| \leq 1$. Thus if $|i-p|>1$, then $X_{i} \notin \mathrm{QL}(Y)$ and all the more $\mathrm{QL}\left(X_{i}\right) \not \supset \mathrm{QL}(Y)$, i.e. $X_{i} \notin Y^{\uparrow}$. 
b) Assume that $X_{p} \in Y^{\uparrow}$ and $X_{q} \in Y^{\uparrow}$ for two indices $p<q$ in $[0, n]$, and that $X_{i} \in$ $\mathrm{QL}(Y)$ for some $i \in[0, n]$. Then $X_{i} \in \mathrm{QL}\left(X_{p}\right)$ and $X_{i} \in \mathrm{QL}\left(X_{q}\right)$ because $\mathrm{QL}(Y) \subset$ $\mathrm{QL}\left(X_{p}\right) \cap \mathrm{QL}\left(X_{q}\right)$. Since $\left(X_{0}, \ldots, X_{n}\right)$ is direct this forces $|i-p| \leq 1$ and $|i-q| \leq 1$. Thus, if $|i-p|>1$ or $|i-q|>1$ then $X_{i} \notin \mathrm{QL}(Y)$, and all the more $\mathrm{QL}(Y) \not \subset \mathrm{QL}\left(X_{i}\right)$, i.e. $X_{i} \notin Y^{\uparrow}$.

c) Given a ray $X_{p} \in Y^{\uparrow}$ we conclude from a) that $X_{i} \in Y^{\uparrow}$ at most for $i=p-1$, $p, p+1$ if $0<p<n$, while for $p=0 X_{i} \in Y^{\uparrow}$ at most for $i=0,1$ and for $p=0$ at most for $i=n-1, n$. Thus $Y^{\uparrow}$ contains either none or one or two adjacent rays in $\left\{X_{0}, \ldots, X_{n}\right\}$.

\section{Definition 9.5.}

(i) Suppose $\left(X_{0}, \ldots, X_{n}\right)$ is a direct QL-path of length $n \geq 1$. We call two adjacent rays $X_{p}, X_{p+1}(0 \leq p<n)$ twins, if $X_{p}^{\downarrow} \cap X_{p+1}^{\downarrow} \neq \emptyset$, and also say that $\left(X_{p}, X_{p+1}\right)$ is a twin pair. We say that a ray $X_{q}(0 \leq q \leq n)$ is a single in the QL-path $\left(X_{0}, \ldots, X_{n}\right)$, if $X_{q}$ is not a twin, i.e., it is not part of a twin pair.

(ii) We call a ray $Y$ an anchor of a ray $X_{i}$ in $\left(X_{0}, \ldots, X_{n}\right)$, if either $X_{i}$ has a twin $X_{j}$ $(|j-i|=1)$ and $Y \in X_{i}^{\downarrow} \cap X_{j}^{\downarrow}$, or $X_{i}$ is a single and $Y \in X_{i}^{\downarrow}$. We then also say that $X_{i}$ is anchored at $Y$.

By Theorem 9.4, a path is single iff $X_{q}^{\downarrow} \cap X_{j}^{\downarrow}=\emptyset$ for every $j$ with $|j-q|=1$, which then holds for all $j \neq q$ in $\{0, \ldots, n\}$.

Procedure 9.6. We choose an ordered set $S=\left(Y_{0}, \ldots, Y_{m}\right)$ of anchors for the direct $Q L$ path $T=\left(X_{0}, \ldots, X_{n}\right)$ such that for each $i \in[0, n]$ the set of chosen anchors $\left(Y_{0}, \ldots, Y_{k}\right)$ for $\left(X_{0}, \ldots, X_{i}\right)$ is as small as possible, and then call $S$ an anchor set of $T$. More precisely we proceed as follows. We choose an anchor $Y_{0}$ of $X_{0}$. If $\left(X_{0}, X_{1}\right)$ is a twin pair, we choose the anchor $Y_{0}$ also for $X_{1}$. Otherwise we choose for $X_{1}$ a new anchor $Y_{1}$. If anchors $\left(Y_{0}, \ldots, Y_{k}\right)$ have been chosen for $\left(X_{0}, \ldots, X_{i}\right), i<n$, we choose for $X_{i+1}$ again the anchor $Y_{k}$, if $\left(X_{i}, X_{i+1}\right)$ is a twin pair and $Y_{k}$ has not already been chosen twice in the anchor list for $\left(X_{0}, \ldots, X_{i}\right)$, which means that $\left(X_{i-1}, X_{i}\right)$ is not a twin pair 8 Otherwise we choose for $X_{i+1}$ a new anchor $Y_{k+1}$. Note that for different rays $X_{i}, X_{j}$ with $i<j$ we have in $S$ anchors $Y_{k}, Y_{\ell}$ with $k \leq \ell$.

In particular we can choose as anchor of a single $X_{q}$ the ray $X_{q}$ itself. An anchor set $S$ arising in this way is called a special anchor set of the direct QL-path $\left(X_{0}, \ldots, X_{n}\right)$.

\section{Remark 9.7.}

a) All anchor sets of $T=\left(X_{0}, \ldots, X_{n}\right)$ have the same length $m$. Moreover $m \leq n \leq 2 m$, where $n=m$ if $S$ contains only singles and $n=2 m$ if $T$ contains only twins, where no two twin pairs have a ray in common.

b) If $\left(Y_{0}, \ldots, Y_{m}\right)$ is an anchor set of $\left(X_{0}, \ldots, X_{n}\right)$, then every tuple of rays $\left(Y_{0}^{\prime}, \ldots, Y_{m}^{\prime}\right)$ with $Y_{k}^{\prime} \leq_{\mathrm{QL}} Y_{k}$ for $0 \leq k \leq m$ is again an anchor set of $\left(X_{0}, \ldots, X_{n}\right)$.

All this is evident from Definition 9.5.

\footnotetext{
${ }^{8}$ cf. $\$ 10$ below about different twin-pairs which are not disjoint.
} 
We turn to the problem of specifying which finite ordered sets $\left(Y_{0}, \ldots, Y_{m}\right)$ of rays in $V$ $(m \geq 1)$ can serve as an anchor set of a direct QL-path. The following fact will be of help.

Lemma 9.8. Assume that $X_{1}, X_{2}, Y_{1}, Y_{2}$ are rays in $V$ with $Y_{1} \in X_{1}^{\downarrow}, Y_{2} \in X_{2}^{\downarrow}$, and that the pair $\left(Y_{1}, Y_{2}\right)$ is quasilinear. Then $\left(X_{1}, X_{2}\right)$ is quasilinear.

Proof. We have $\mathrm{QL}\left(Y_{1}\right) \subset \mathrm{QL}\left(X_{1}\right), \mathrm{QL}\left(Y_{2}\right) \subset \mathrm{QL}\left(X_{2}\right)$, and $Y_{2} \in \mathrm{QL}\left(Y_{1}\right)$. From this we conclude that $Y_{2} \in \mathrm{QL}\left(X_{1}\right)$, and then that $X_{1} \in \mathrm{QL}\left(Y_{2}\right) \subset \mathrm{QL}\left(X_{2}\right)$, which proves that $\left(X_{1}, X_{2}\right)$ is quasilinear.

Definition 9.9. We call a pair $\left(Y_{1}, Y_{2}\right)$ of rays in $V$ subquasilinear, abbreviated sql, if there exists a quasilinear pair $\left(X_{1}, X_{2}\right)$ with $Y_{1} \in X_{1}^{\downarrow}, Y_{2} \in X_{2}^{\downarrow}$, and say that the QL-pair $\left(X_{1}, X_{2}\right)$ covers $\left(Y_{1}, Y_{2}\right)$. We call a tuple of rays $\left(Y_{0}, \ldots, Y_{m}\right), m \geq 1$, subquasilinear, if $\left(Y_{i}, Y_{i+1}\right)$ is sql for $0 \leq i<m$, and say that $\left(Y_{0}, \ldots, Y_{m}\right)$ is a subquasilinear sequence of length $m$. Finally, we say that an sql sequence $\left(Y_{0}, \ldots, Y_{m}\right)$ is direct, if for any rays $Y_{k}, Y_{\ell}$ with $|k-\ell|>1$ in the sequence the pair $\left(Y_{k}, Y_{\ell}\right)$ is not quasilinear.

Theorem 9.10. Every anchor set $S=\left\{Y_{0}, \ldots, Y_{m}\right\}$ of a direct quasilinear path $\left(X_{0}, \ldots, X_{n}\right)$ is a direct subquasilinear sequence.

Proof. Let $Y_{k}$ and $Y_{\ell}$ be different rays in $S$ with $k<\ell$, and let $X_{i}$ and $X_{j}$ be rays in $\left(X_{0}, \ldots, X_{n}\right)$ which are anchored at $Y_{k}$ and $Y_{\ell}$ respectively, $i<j$. Thus $Y_{k} \in X_{i}^{\downarrow}$ and $Y_{\ell} \in X_{j}^{\downarrow}$. If $j>i+1$, then $\left(X_{i}, X_{j}\right)$ is not quasilinear, since the QL-path $\left(X_{0}, \ldots, X_{n}\right)$ is direct. It follows by Lemma 9.8 that $\left(Y_{k}, Y_{\ell}\right)$ is not quasilinear. Assume now that $j=i+1$. Then it is clear by Procedure 9.6 that $\ell=k+1$. Since the QL-pair $\left(X_{i}, X_{i+1}\right)$ covers that pair $\left(Y_{k}, Y_{k+1}\right)$, evidently, $\left(Y_{0}, \ldots, Y_{m}\right)$ is a direct subquasilinear sequence.

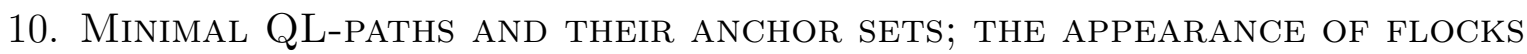

Our first topic in this section is the case that in a direct QL-path there exist adjacent twin pairs, which are not disjoint.

Definition 10.1. We call a subsequence $\left(X_{p}, \ldots, X_{q}\right)$ of a direct $Q L$-path $T=\left(X_{0}, \ldots, X_{n}\right)$ with $q-p \geq 2$ a flock in $T$, if $\left(X_{i}, X_{i+1}\right)$ is a twin pair in $T$ for every $i$ in $[p, q-1]$, and so $T$ has an anchor set $S=\left(Y_{0}, \ldots, Y_{m}\right)$, in which these twin pairs have anchors $Y_{k}, Y_{k+1}, \ldots, Y_{k+t}$ for some $k \in[0, m]$ and $t=q-p-1$. In other terms, we have a diagram

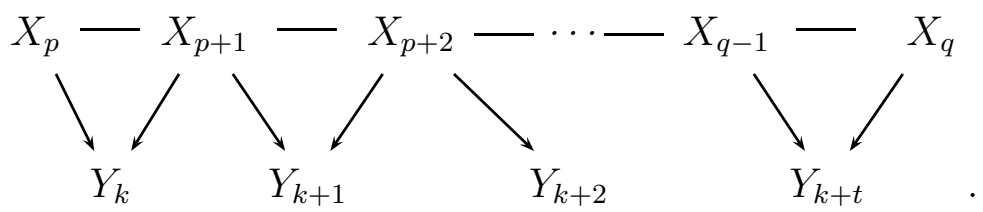

We call $q-p=t+1$ the length of the flock $\left(X_{p}, \ldots, X_{q}\right)$. We further call a twin pair in $T$, which is not a member of a flock, an isolated twin pair.

\section{Remark 10.2.}

a) In a flock, as seen in diagram (10.1), the common ray of any two adjacent twin pairs has two anchors in the sequence $S$, while each ray in an isolated twin pair has only one anchor in $S$. 
b) None of the adjacent pairs $\left(Y_{k+i-1}, Y_{k+i}\right)$ in (10.1) is quasilinear (but, of course, is sql), since otherwise the QL-path $\left(X_{p}, \ldots, X_{q}\right)$ would not be direct. Indeed, if, say, $\left(Y_{k}, Y_{k+1}\right)$ would be $q$, then by Lemma 9.8 the pair $\left(X_{p}, X_{p+2}\right)$ would be ql.

c) For the same reason it cannot happen in $S$, that two non-adjacent rays form a quasilinear pair, as already stated in Theorem 9.10.

Lemma 10.3. Assume that $T=\left(X_{0}, \ldots, X_{n}\right)$ is a $Q L$-path, and that for given $p, q \in[0, n]$ with $p+1<q$ the sets $\left(X_{p}^{\downarrow}\right)^{\uparrow}$ and $\left(X_{q}^{\downarrow}\right)^{\uparrow}$ are not disjoint. We choose $Y_{1} \in X_{p}^{\downarrow}, Y_{2} \in X_{q}^{\downarrow}$ such that there is some $Z \in Y_{1}^{\uparrow} \cap Y_{2}^{\uparrow}$.

a) Then we have a $Q L$-path $\left(X_{0}, \ldots, X_{p}, Z, X_{q}, \ldots, X_{n}\right)$ with a diagram

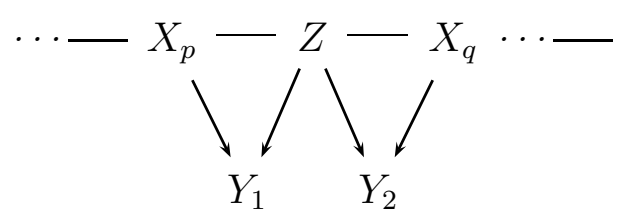

b) If the new path $T^{\prime}=\left(X_{0}, \ldots, X_{p}, Z, X_{q}, \ldots, X_{n}\right)$ is direct, then $\left(X_{p}, Z\right)$ and $\left(Z, X_{q}\right)$ are twin pairs, and so $\left(X_{p}, Z, X_{q}\right)$ is a flock of length 2 in $T^{\prime}$.

c) If $q=p+2$, then $T^{\prime}$ has again length $n$; otherwise $T^{\prime}$ is shorter.

Proof. The pairs $\left(X_{p}, Z\right)$ and $\left(Z, X_{q}\right)$ are quasilinear, since $Y_{1}^{\uparrow}$ and $Y_{2}^{\uparrow}$ are convex quasilinear sets (Theorem 5.6). Thus $T^{\prime}$ is indeed a quasilinear path. Now assertions b) and c) are immediate by Definition 10.1 and an easy counting.

The crux in this lemma is that, even if we assume that $T$ is direct, in general there is no apparent way to decide whether $T^{\prime}$ is direct or not.

Definition 10.4. We call a QL-path $\left(X_{0}, \ldots, X_{n}\right)$ minimal, if there does not exist a $Q L$ path from $X_{0}$ to $X_{n}$ of length $<n$.

If in Lemma 16.3 the path $T$ is minimal, then $T^{\prime}$ is again minimal (and $q=p+2$ ), and so $T^{\prime}$ is certainly direct.

Theorem 10.5. Assume that $T=\left(X_{0}, \ldots, X_{n}\right)$ is a minimal $Q L$-path of length $n \geq 2$, and $S=\left(Y_{0}, \ldots, Y_{m}\right)$ is an anchor set of $T$. Assume further that $\left(Y_{k}, \ldots, Y_{k+t+1}\right)$ is a maximal subsequence of $S$ with $t \geq 0, k \geq 0$, and $k+t<m$, such that

$$
Y_{i}^{\uparrow} \cap Y_{i+1}^{\uparrow} \neq \emptyset \quad \text { for } k \leq i \leq k+t .
$$

Choose rays $X_{s}$ and $X_{r}$ in $T$ such that $Y_{k}$ is an anchor of $X_{s}$ and $Y_{k+t+1}$ an anchor of $X_{r}$ in $S$. Finally choose rays $W_{i} \in Y_{i}^{\uparrow} \cap Y_{i+1}^{\uparrow}$ for $k \leq i \leq k+t$. Then

$$
T^{\prime}:=\left(X_{0}, \ldots, X_{s}, W_{k}, \ldots, W_{k+t}, X_{r}, \ldots, X_{n}\right)
$$

is again a minimal QL-path of length $n$ which admits $S$ as anchor set. The sequence $\left(X_{s}, W_{k}, \ldots, W_{k+t}, X_{r}\right)$ is a flock in $T$ of length $t+2$ with anchors $Y_{k}, \ldots, Y_{k+t+1}$. This flock is maximal in $T^{\prime}$, i.e., there is no flock of length $>t+2$ in $T^{\prime}$ which contains $\left(X_{s}, W_{k}, \ldots, W_{k+t}, X_{r}\right)$. 
Proof. Using Lemma 10.3 iteratively, we obtain a diagram

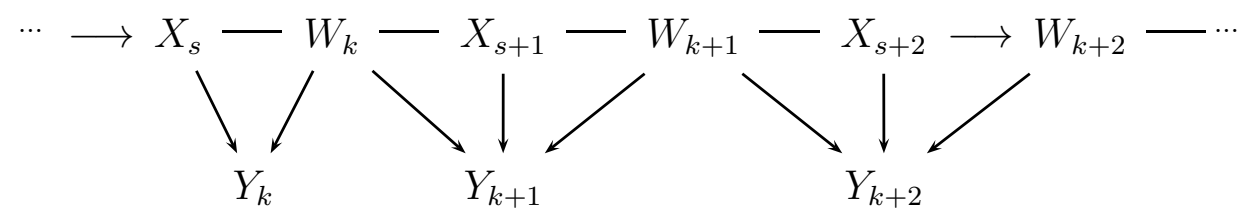

The upper row is a QL-path, in which the pairs $\left(W_{k}, W_{k+1}\right),\left(W_{k+1}, W_{k+2}\right), \ldots$ are quasilinear, since they are contained in the convex quasilinear sets $Y_{k+1}^{\uparrow}, Y_{k+2}^{\uparrow}, \ldots$ Thus $(*)$ can be reduced to a diagram

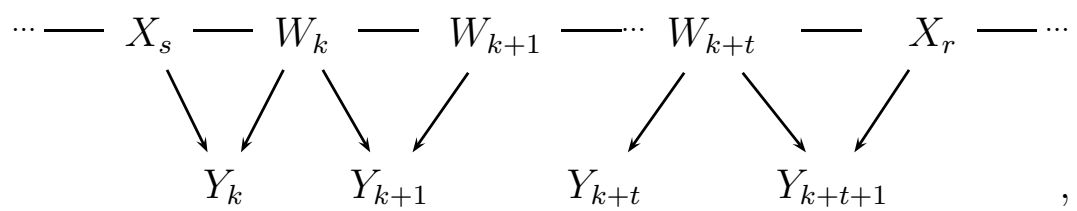

where to the left of $W_{k}$ and $Y_{k}$, and to the right of $W_{k+t}$ and $Y_{k+t}$ there appear parts of the anchor diagram of $T$ over $S$. In the upper row of (10.3) we have a QL-path

$$
T^{\prime}:=\left(X_{0}, \ldots, X_{s}, W_{k}, \ldots, W_{k+t}, X_{r}, \ldots, X_{n}\right)
$$

of length $\leq n$ starting at $X_{0}$ and ending at $X_{n}$, as does $T$. Thus $T^{\prime}$ is minimal, whence direct, so that we can speak about twin pairs and flocks in $T^{\prime}$, and $T^{\prime}$ has again length $n$.

From diagram (10.3) we read off that $\left(X_{s}, W_{k}, \ldots, W_{k+t}, X_{r}\right)$ is a flock in $T^{\prime}$ of length $t+2$. Since $Y_{k-1}^{\uparrow} \cap Y_{k}^{\uparrow}=\emptyset$ and $Y_{k+t+1}^{\uparrow} \cap Y_{k+t+2}^{\uparrow}=\emptyset$, it is evident that $Y_{k-1}$ and $Y_{k+t+2}$ are not anchors of $X_{s}$ and $X_{r}$ respectively. It follows that $T^{\prime}$ admits $S$ as an anchor set 9 and then, that the flock $\left(X_{s}, W_{k}, \ldots, W_{k+t}, X_{r}\right)$ is maximal in $T^{\prime}$.

Exploring properties of anchor sets beyond Theorem 9.10, we take a closer look at Procedure 9.6 to obtain such sets.

Definition 10.6. Assume that $T=\left(X_{0}, \ldots, X_{n}\right)$ is a direct $Q L$-path and $S=\left(Y_{0}, \ldots, Y_{n}\right)$ is an anchor set of $T$. If $X_{i}$ is a ray in $T$, we call the ray $Y_{k}$ chosen for $X_{i}$ as anchor in $S$ the legal anchor of $X_{i}$ in $S$. The other anchors of $X_{i}$ in $S$ are named illegal anchors.

(In fact $X_{i}$ can have at most one illegal anchor in $S$, see below).

Looking at Procedure 9.6, the following is now easily verified.

\section{Scholium 10.7.}

a) $A$ single $X_{i}$ in $T$ has only a legal anchor in $S$.

b) Both rays in an isolated twin pair $\left(X_{i}, X_{i+1}\right)$ have one common legal anchor in $S$, and no illegal anchors.

c) If $\left(X_{p}, X_{p+1}, \ldots, X_{q}\right)$ is a maximal flock in $T, q=p+t+1$ with $t \geq 0$, then each interior ray $X_{i}, p<i<q$, of the flock has one legal and one illegal anchor in $S$, while the border rays $X_{p}$ and $X_{q}$ have only legal anchors. These are $Y_{k}$ and $Y_{k+t+1}$. The legal anchors of $X_{p+1}, \ldots, X_{p+t}$ are $Y_{k}, \ldots, Y_{k+t}$, while the illegal anchors of these rays are $Y_{k+1}, \ldots, Y_{k+t+1}$.

d) It follows that, if a ray $X_{i}$ has a legal anchor $Y_{u}$ and an illegal anchor $Y_{w}$ in $S$, then $w=u+1$.

\footnotetext{
${ }^{9}$ Recall Procedure 9.6 .
} 
Theorem 10.8. Assume that $S=\left(Y_{0}, \ldots, Y_{m}\right)$ is an anchor set of a direct QL-path $T=$ $\left(X_{0}, \ldots, X_{n}\right)$. Then the downsets $Y_{0}^{\downarrow}, \ldots, Y_{m}^{\downarrow}$ are pairwise disjoint.

Proof. Given rays $Y_{k}$ and $Y_{\ell}$ with $k<\ell$, suppose that there exists a ray $Z \in Y_{k}^{\downarrow} \cap Y_{\ell}^{\downarrow}$. We pick rays $X_{p}$ and $X_{q}$ in $T$, with legal anchors $Y_{k}$ and $Y_{\ell}$, respectively. Then $p<q$ and

$$
X_{p} \in Y_{k}^{\uparrow} \subset Z^{\uparrow}, \quad X_{q} \in Y_{\ell}^{\uparrow} \subset Z^{\uparrow} .
$$

Thus $Z^{\uparrow}$ meets $T$ in two rays $X_{p}$ and $X_{q}$. So $q=p+1$ and $\left(X_{p}, X_{q}\right)$ is a twin-pair with common anchor $Z$. This forces $\ell=k+1$. We infer from Scholium 10.7, b that the twin pair $\left(X_{p}, X_{p+1}\right)$ is not isolated in $T$, and thus is part of a maximal flock in $T$ which either extends to the left of $X_{p}$ or to the right of $X_{p+1}$ (or both). Thus we have a diagram

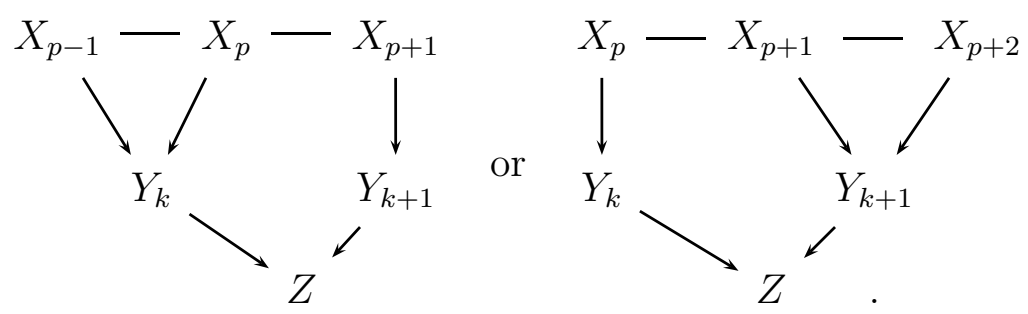

Both diagrams cannot exist, since then $Z^{\uparrow}$ would contain at least three rays of $T$, contradicting Theorem 9.10, Thus $Y_{k}^{\downarrow}$ and $Y_{\ell}^{\downarrow}$ are disjoint.

In a similar way we obtain a result about the upsets $Y_{k}^{\uparrow}$.

Theorem 10.9. Assume again that $S=\left(Y_{0}, \ldots, Y_{m}\right)$ is an anchor set of a direct QL-path $T=\left(X_{0}, \ldots, X_{n}\right)$. Given rays $Y_{k}, Y_{\ell}$ in $S$ with $k<\ell$, the intersection $Y_{k}^{\uparrow} \cap Y_{\ell}^{\uparrow}$ contains at most one ray of $T$, and in this case $\ell=k+1$.

Proof. Suppose that there exist two rays $X_{p}, X_{q}, p<q$, in $S$ which both are in $Y_{k}^{\uparrow} \cap Y_{\ell}^{\uparrow}$. Since $Y_{k}^{\uparrow}$ meets $S$ in two rays $X_{p}, X_{q}$ it is evident (cf. Theorem 9.4), that $q=p+1$ and $\left(X_{p}, X_{q}\right)$ is a twin-pair. This forces $\ell=k+1$. Both $Y_{k}$ and $Y_{k+1}$ are common anchors of $X_{p}$ and $X_{p+1}$ in $S$. We conclude from Scholium 10.7, that $Y_{k}$ is the legal anchor of both $X_{p}$ and $X_{p+1}$, while $Y_{k+1}$ is an illegal anchor of both $X_{p}$ and $X_{p+1}$, and then, that $X_{p}$ and $X_{p+1}$ are interior rays of a maximal flock in $T$. Thus we have a diagram

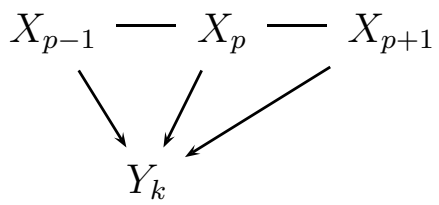

and an analogous diagram involving $X_{p}, X_{p+1}, X_{p+2}, Y_{k+1}$. But such diagrams do not exist, since $Y_{k}^{\uparrow}$, and also $Y_{k+1}^{\uparrow}$, can meet $T$ in at most 2 rays (cf. Theorem 9.4). We conclude that $Y_{k}^{\uparrow} \cap Y_{k+1}^{\uparrow}$ contains at most one ray of $T$.

Definition 10.10. We call a pair $(T, S)$ consisting of a direct $Q L$-path $T=\left(X_{0}, \ldots, X_{n}\right)$ and an anchor set $S=\left(Y_{0}, \ldots, Y_{m}\right)$ of $T$ flocky, if for any $\operatorname{pair}\left(Y_{i}, Y_{i+1}\right)$ in $S$ with $Y_{i}^{\uparrow} \cap Y_{i+1}^{\uparrow} \neq \emptyset$ there exists a ray $W$ in $T$ with $W \in Y_{i}^{\uparrow} \cap Y_{i+1}^{\uparrow}$.

Note that then $W$ is the unique such ray, as stated in Theorem 10.9.

We have an explicit description of all maximal flocks in a flocky pair as follows. 
Theorem 10.11. Assume that $(T, S)$ is a flocky pair, $T=\left(X_{0}, \ldots, X_{n}\right), S=\left(Y_{0}, \ldots, Y_{m}\right)$. Assume further that $\left(Y_{k}, \ldots, Y_{k+t+1}\right)$ is a maximal subsequence of $S$ with

$$
t \geq 0, k \geq 0, k+t<m, Y_{i}^{\uparrow} \cap Y_{i+1}^{\uparrow} \neq \emptyset \quad \text { for } \quad k \leq i \leq k+t .
$$

Let $W_{i}$ denote the unique ray of $T$ contained in $Y_{i}^{\uparrow} \cap Y_{i+1}^{\uparrow}(k \leq i \leq k+t)$. We have $W_{k}=X_{p}$, $W_{k+t}=X_{q}$ with indices $p<q$ in $[0, n]$.

a) The sequence $\left(W_{k}, \ldots, W_{k+t}\right)$ coincides with the subpath $\left(X_{p}, \ldots, X_{q}\right)$ of $T$, in particular $q=p+t$, and this is a maximal subpath of $T$ with the property that each of its rays has two anchors in $S$. These are the legal anchors $Y_{k}, \ldots, Y_{k+t}$ and the illegal anchors $Y_{k+1}, \ldots, Y_{k+t+1}$. The subpath $\left(X_{p}, \ldots, X_{q}\right)$ is a flock in $T$, perhaps not a maximal flock.

b) Assume that $p>0$ and $q=p+t<n$. Then the unique anchor of $X_{p-1}$ in $S$ is either $Y_{k}$ or $Y_{k-1}$, and the unique anchor of $Y_{q+1}$ in $S$ is either $Y_{k+t}$ or $Y_{k+t+1}$. If $X_{p-1}$ and $Y_{q+1}$ have the anchors $Y_{k}$ and $Y_{k+t}$, then the maximal flock containing $\left(X_{p}, \ldots, X_{q}\right)$ is $\left(X_{p-1}, \ldots, X_{q+1}\right)$ while in the other cases we have to delete either $X_{p-1}$ or $X_{q+1}$ or both in the subpath $\left(X_{p-1}, \ldots, X_{q+1}\right)$ to obtain a maximal flock.

c) In the case $p=0, q<n$, we have the maximal flock $\left(X_{0}, \ldots, X_{q+1}\right)$ if $X_{q+1}$ has anchor $Y_{k+t}$, and $\left(X_{0}, \ldots, X_{q}\right)$ if $X_{q+1}$ has anchor $Y_{k+t+1}$. In the case $p>0, q=n$ we have the maximal flock $\left(X_{p-1}, \ldots, X_{n}\right)$ if $X_{p-1}$ has anchor $Y_{k}$, and $\left(X_{p}, \ldots, X_{n}\right)$ if $X_{p-1}$ has anchor $Y_{k-1}$. In the trivial case $p=0, q=n$, the path $T$ itself is a flock in $T$.

Proof. We focus on the case $p>0, q<n$. Every pair $\left(W_{i}, W_{i+1}\right), k \leq i \leq k+t$ is quasilinear since both $W_{i}$ and $W_{i+1}$ are rays in the convex quasilinear set $Y_{i+1}^{\uparrow}$. Thus $\left(W_{k}, \ldots, W_{k+t}\right)$ is a QL-path. All $W_{i}$ are rays in the QL-path $\left(X_{p}, \ldots, X_{q}\right)$. Since this QL-path is direct, a pair $\left(X_{i}, X_{j}\right)$ with $p \leq i<j \leq q$ can be quasilinear only if $j=i+1$. This forces

$$
\left(W_{k}, \ldots, W_{k+t}\right)=\left(X_{p}, \ldots, X_{q}\right)
$$

and $q=p+t$. Obviously each $W_{i}$ has in $S$ two anchors, the legal anchor $Y_{i}$ and the illegal anchor $Y_{i+1}$, and $\left(W_{k}, \ldots, W_{k+t}\right)$ is a flock in $T$. $X_{p-1}$ cannot have two anchors in $S$, since these would be $Y_{k-1}$ and $Y_{k}$ (cf. Scholium 10.7), contradicting the maximality of the family $\left(Y_{k}, \ldots, Y_{k+t+1}\right)$ with (10.4). Same for $X_{q+1}$. Thus the path $\left(X_{p}, \ldots, X_{q}\right)$ is maximal in $T$ with the property, that each of its rays has two anchors in $S$. If $X_{p-1}$ has the anchor $Y_{k}$ then $\left(X_{p-1}, X_{p}\right)$ is a twin pair with anchor $Y_{k}$. If $X_{p-1}$ has the (only) anchor $Y_{k-1}$, then $\left(X_{p-1}, X_{p}\right)$ is not a twin pair. Thus is the first case $\left(X_{p-1}, \ldots, X_{q}\right)$ is a flock, but in the second case not. Analogously $\left(X_{p}, \ldots, X_{q+1}\right)$ is a flock if and only if $X_{q+1}$ has the anchor $Y_{k+t}$. This proves all claims in the case $p>0, q<n$. In the cases $p=0, q<n$ and $p>0, q=n$ the same arguments work, where only the rays $X_{q+1}$ and $X_{p-1}$, respectively, should be taken care of.

How much does the appearance of flock depend on the choice of the anchor set $S$ of $T$ ? In preparation for answering this question, we need an important general fact.

Proposition 10.12. Assume that $T=\left(X_{0}, \ldots, X_{n}\right)$ is a direct QL-path and that $S=$ $\left(Y_{0}, \ldots, Y_{m}\right), S^{\prime}=\left(Y_{0}^{\prime}, \ldots, Y_{m^{\prime}}^{\prime}\right)$ are two anchor sets of $T$. Then $m=m^{\prime}$ and for any ray $X_{p}$ 
in $T$ the anchors of $X_{p}$ in $S$ correspond uniquely to the anchors of $X_{p}$ in $S^{\prime}$. More precisely, if $Y_{k}$ is a legal (resp. illegal) anchor of $X_{p}$ in $S$, then $Y_{k}^{\prime}$ is a legal (resp. illegal) anchor of $X_{p}$ in $S^{\prime}$.

Proof. Using Procedure 9.6, we obtain step by step anchor sets $\left(Y_{0}, \ldots, Y_{m(i)}\right)$ of the subpaths $\left(X_{0}, \ldots, X_{i}\right), 0 \leq i \leq n$, with $\{0\}=m(0) \leq m(1) \leq \cdots \leq m(n)=m$, where the chosen anchors of a ray $X_{p}$ with $p \leq i$ remain the same when proceeding from $\left(X_{0}, \ldots, X_{i}\right)$ to $\left(X_{0}, \ldots, X_{i+1}\right)$. Applying this procedure a second time, we obtain anchor sets $\left(Y_{0}^{\prime}, \ldots, Y_{m(i)}^{\prime}\right)$, $0 \leq i \leq n$ of the same lengths $m(i)$. By induction on $i$ for $i=0, \ldots, n$ we see that the last claim of the proposition holds for all rays $X_{p}$ with $p \leq i$, in $\left(Y_{0}, \ldots, Y_{m(i)}\right)$ and $\left(Y_{0}^{\prime}, \ldots, Y_{m(i)}^{\prime}\right)$, and thus for all rays $X_{p}$ in $T$.

Comment. This uniqueness result for anchor sets is less trivial than it may appear at first glance. Recall that a point in the down set $X^{\downarrow}$ of a ray $X$ means a QL-star contained in $\mathrm{QL}(X)$. There can be many such stars for fixed $X$ which are widely unrelated.

Proposition 10.13. Given a direct $Q L$-path $T=\left(X_{0}, \ldots, X_{n}\right)$ and an anchor $\left(Y_{0}, \ldots, Y_{m}\right)$ of $T$, if $Y_{k} \in X_{p}^{\downarrow}$ for some $k \in[0, m], p \in[0, n]$, then $Y_{k}$ is an anchor of $X_{p}$ (Definition 9.5. (ii)).

Proof. We choose $q \in[0, n]$ such that $Y_{k}$ is the legal anchor of $X_{q}$. Then $X_{p}$ and $X_{q}$ are in $Y_{k}^{\uparrow}$, and thus $\left(X_{p}, X_{q}\right)$ is quasilinear by Theorem 5.6, whence $q \in\{p-1, p+1\}$. If $q=p$ then $X_{p}$ is the legal anchor of $Y_{k}$. If $q=p-1$ then $X_{p-1}$ and $X_{p}$ are in $Y_{k}^{\uparrow}$. Thus $\left(X_{p-1}, X_{p}\right)$ is a twin-pair in $T$, with anchor $Y_{k}$, and so $Y_{k}$ is an anchor of $X_{p}$. If $q=p+1$ we conclude in the same way that $\left(X_{p}, X_{p+1}\right)$ is a twin pair, and so $Y_{k}$ is an anchor of $X_{p}$.

Corollary 10.14. Assume that $\left(Y_{0}, \ldots, Y_{m}\right)$ and $\left(Y_{0}^{\prime}, \ldots, Y_{m}^{\prime}\right)$ are anchor sets of a direct QL-path $\left(X_{0}, \ldots, X_{n}\right)$. Let $p \in[0, n]$ and $k \in[0, m]$. Then $Y_{k} \in X_{p}^{\downarrow}$ iff $Y_{k}^{\prime} \in X_{p}^{\downarrow}$.

Proof. Immediate from Propositions 10.12 and 10.13.

Theorem 10.15. Assume again that $S=\left(Y_{0}, \ldots, Y_{m}\right)$ and $S^{\prime}=\left(Y_{0}^{\prime}, \ldots, Y_{m}^{\prime}\right)$ are anchor sets of a direct $Q L$-path $T=\left(X_{0}, \ldots, X_{n}\right)$. Assume further that the pair $(T, S)$ is flocky. Then $\left(T, S^{\prime}\right)$ is flocky.

Proof. Given $k \in[0, m-1]$ such that $Y_{k}^{\uparrow} \cap Y_{k+1}^{\uparrow} \neq \emptyset$ there is some $p \in[0, n]$ with $X_{p} \in$ $Y_{k}^{\uparrow} \cap Y_{k+1}^{\uparrow}$, since $(T, S)$ is flocky. We conclude by Corollary 10.14 that $X_{p} \in\left(Y_{k}^{\prime}\right)^{\uparrow} \cap\left(Y_{k+1}^{\prime}\right)^{\uparrow}$. Thus $\left(T, S^{\prime}\right)$ is flocky.

Definition 10.16. In consequence of this theorem, a QL-path $T$ is named a flocky direct QL-path, if $(T, S)$ is flocky for any anchor set $S$ of $T$.

Theorem 10.5 leads to a procedure that turns a minimal QL-path to a flocky minimal path.

Definition 10.17. Assume that $S=\left(Y_{0}, \ldots, Y_{m}\right)$ is a subquasilinear sequence (cf. Definition 9.9).

(i) A track in $S$ is a subsequence $\left(Y_{k}, Y_{k+1}, \ldots, Y_{k+t+1}\right)$ with $t \geq 0, k+t<m$, such that $Y_{i}^{\uparrow} \cap Y_{i+1}^{\uparrow} \neq \emptyset$ for $k \leq i \leq k+t$. 
(ii) A subsequence $A$ of $S$ is called trackless, if no ray in $A$ is a member of a track in $S$. Note that $S$ is the disjoint union of its maximal tracks and its maximal trackless subsequences.

In this terminology Theorem 10.5 states that, given a maximal track $U=\left(Y_{k}, \ldots, Y_{k+t+1}\right)$ in an anchor set $S=\left(Y_{0}, \ldots, Y_{m}\right)$ of a minimal QL-path $T=\left(X_{0}, \ldots, X_{n}\right)$, after choosing rays $W_{i}$ in $Y_{i}^{\uparrow} \cap Y_{i+1}^{\uparrow}$ for $k \leq i \leq t$, we obtain a new minimal QL-path

$$
T^{\prime}=\left(X_{0}, \ldots, X_{s}, W_{k}, \ldots, W_{k+t}, X_{r}, \ldots, X_{n}\right),
$$

again of length $n$, which also has $S$ as an anchor set. If $k>0$, then $X_{s}$ has the anchor $Y_{k}$ or $Y_{k-1}$ in $S$, but not both, since otherwise the track $U$ would not be maximal. If $k+t<m$, then for the same reason $X_{r}$ has the anchor $Y_{k+t}$ or $Y_{k+t+1}$, but not both. If $k=0$, then $s=0$, and of course, $Y_{0}$ is the unique anchor of $X_{0}$ in $S$, while if $k+t=m$ we have $r=n$, and $Y_{m}$ is the unique anchor of $X_{n}$.

It is now immediate from Theorem 10.11 that the subpath

$$
\Pi=\left(X_{s}, W_{k}, \ldots, W_{k+t}, X_{r}\right)
$$

of $T^{\prime}$ is flocky with anchor set $U$ and has a unique maximal flock. This flock is $\Pi$ itself in the case that $X_{s}$ has anchor $Y_{k}$ and $X_{r}$ has anchor $Y_{k+t}$. Otherwise the maximal flock of $\Pi$ is obtained by omitting in $\Pi$ the ray $X_{s}$ or the ray $X_{r}$ or both.

Definition 10.18. We call $T^{\prime}$ a flock modification of the minimal QL-path $T$ on the track $U$. Modifying $T$ successively on all maximal tracks in $S$ we obtain a flocky minimal $Q L$-path $\widehat{T}$ of length $n$ with anchor set $S$, which we call a total flock modification of $T$.

Remark 10.19. Given a flock modification $T^{\prime}=\left(X_{0}, \ldots, X_{s}, W_{k}, \ldots, W_{k+t}, X_{r}, \ldots, X_{n}\right)$ of $T$ on the track $U=\left(Y_{k}, \ldots, Y_{k+t+1}\right)$, we obtain all flock modifications of $T$ on $U$ by varying each ray $W_{i}, k \leq i \leq k+t$, within $Y_{i}^{\uparrow} \cap Y_{i+1}^{\uparrow}$. The point here is that the sets $Y_{i}^{\uparrow} \cap Y_{i+1}^{\uparrow}$ are convex and quasilinear in $\operatorname{Ray}(V)$.

\section{Further observations on minimal QL-PATHS AND THEIR ANCHOR SETS}

Arguing similarly as often in $\$ 8-910$, we obtain the following facts about minimal QLpaths, mainly by exploiting Theorem 5.6 .

Theorem 11.1. Assume that $\left(X_{0}, \ldots, X_{n}\right)$ is a minimal QL-path.

a) If $p, q \in[0, n]$ and $p+1<q$, then the sets $\left(X_{p}^{\downarrow}\right)^{\uparrow}$ and $\left(X_{q}^{\downarrow}\right)^{\uparrow}$ are disjoint.

b) If $p, q \in[0, n]$ and $p+1=q$, the sets $\left(X_{p}^{\downarrow}\right)^{\uparrow}$ and $X_{q}^{\uparrow}$ are disjoint, and the sets $X_{p}^{\uparrow}$ and $\left(X_{q}^{\downarrow}\right)^{\uparrow}$ as well.

Proof. a): Let $0 \leq p<q \leq n$. Assume that there exists a ray $Z$ in $\left(X_{p}^{\downarrow}\right)^{\uparrow} \cap\left(X_{q}^{\downarrow}\right)^{\uparrow}$. We choose $Y_{1} \in X_{p}^{\downarrow}$ and $Y_{2} \in X_{q}^{\downarrow}$ with $Z \in Y_{1}^{\uparrow} \cap Y_{2}^{\uparrow}$. Then we have the diagram

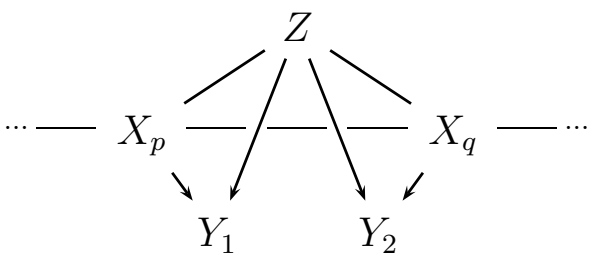


and obtain a QL-path $\left(X_{0}, \ldots, X_{p}, Z, X_{q}, \ldots, X_{n}\right)$ which will be shorter than $\left(X_{0}, \ldots, X_{n}\right)$ if $q>p+1$. Thus $Z$ does not exist in this case, which proves that $\left(X_{p}^{\downarrow}\right)^{\uparrow}$ and $\left(X_{q}^{\downarrow}\right)^{\uparrow}$ are disjoint for $q>p+1$.

b): Let again $0<p<q \leq n$. Assume that there exists some $Z \in\left(X_{p}^{\downarrow}\right)^{\uparrow} \cap X_{q}^{\uparrow}$. Then we have a diagram

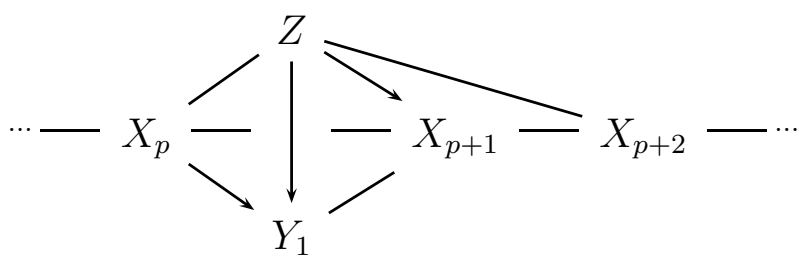

due to the fact that $X_{p+2} \in \mathrm{QL}\left(X_{p+1}\right) \subset \mathrm{QL}(Z)$. But this is impossible since $\left(X_{0}, \ldots, X_{p}, Z, X_{p+2}, \ldots, X_{n}\right)$ would be a QL-path of length $n-1$. We conclude that $\left(X_{p}^{\downarrow}\right)^{\uparrow}$ and $X_{q}^{\uparrow}$ are disjoint. Switching to the opposite path $\left(X_{n}, \ldots, X_{0}\right)$, which again is minimal, we obtain that $X_{p}^{\uparrow}$ and $\left(X_{q}^{\downarrow}\right)^{\uparrow}$ are disjoint.

Given a direct QL-path $T=\left(X_{0}, \ldots, X_{n}\right)$ and an anchor set $S=\left(Y_{0}, \ldots, Y_{m}\right)$ of $T$, we call the full subdiagram of $\hat{\Gamma}_{\mathrm{QL}}(V, q)$ spanned by the rays $\left(X_{0}, \ldots, X_{n}, Y_{0}, \ldots, Y_{m}\right)$ the anchor diagram of the pair $(T, S)$. Most often we display the anchor diagram as a "bipartite" subdiagram of $\hat{\Gamma}_{\mathrm{QL}}(V, q)$, with an upper horizontal row containing the rays of $T$ and a lower horizontal row containing the rays of $S$. In the lower horizontal there do not occur any arrows $(\rightarrow)$ in consequence of Theorem 10.8. But there can be edges $(-)$ and so QL-subpaths of the sql sequence $\left(Y_{0}, \ldots, Y_{n}\right)$, at which we now take a look. First note that all QL-subpaths of $S$ are direct, as stated in Theorem 9.10 .

Lemma 11.2. Assume that $T=\left(X_{0}, \ldots, X_{n}\right)$ is a direct $Q L$-path and $S=\left(Y_{0}, \ldots, Y_{m}\right)$ is an anchor set of $T$. Assume further that $\left(X_{p}, X_{p+1}\right)$ is a twin pair with anchor $Y_{k}$ in $S$. Then neither $\left(Y_{k-1}, Y_{k}\right)$ (if $\left.k>0\right)$ nor $\left(Y_{k}, Y_{k+1}\right)($ if $k<m)$ is $q l$.

Proof. Otherwise we have a diagram

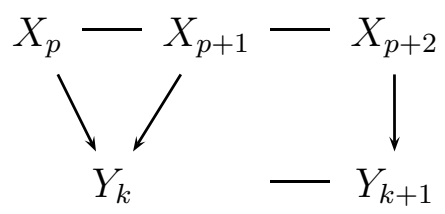

or

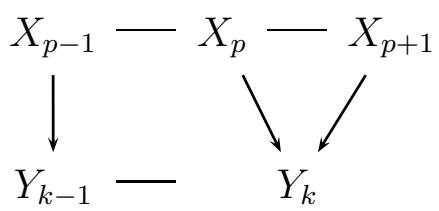

But $(*)$ would imply that $\left(X_{p}, X_{p+2}\right)$ is ql, and $(* *)$ would imply that $\left(X_{p-1}, X_{p+1}\right)$ is $\mathrm{ql}$, in contradiction to the assumption that $T$ is direct.

The lemma has the following immediate consequence

Theorem 11.3. Assume again that $T=\left(X_{0}, \ldots, X_{n}\right)$ is a direct $Q L$-path and $S=\left(Y_{0}, \ldots, Y_{m}\right)$ is a anchor set of $T$. Assume further that a subsequence $\left(Y_{k}, Y_{k+1}, \ldots, Y_{k+t}\right)$ of $T$ with $k \geq 0$, 
$k+t \leq m, t \geq 1$, is a QL-path, necessarily direct. Then the part of the anchor diagram of $(T, S)$ lying over this sequence is an enlargement

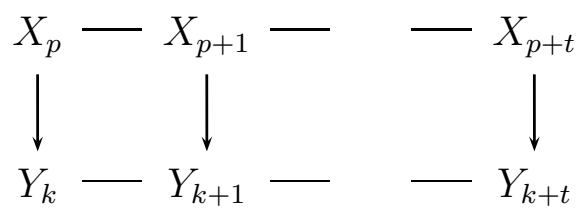

of this subsequence, consisting of singles $X_{p}, X_{p+1}, \ldots, X_{p+t}$ of $T$, and their (legal) anchors $Y_{k}, Y_{k+1}, \ldots, Y_{k+t}$.

We call (11.3) a QL-block (of length $t$ ) in the anchor diagram of $(T, S)$.

In contrast to the flocks in $T$, the subpaths of $T$ appearing as the upper horizontal of a QL-block strongly depend on the choice of the anchor set $S$ of $T$. In particular we can choose for every single $X_{p}$ in $T$ as an anchor the ray $X_{p}$ itself, and then obtain a special anchor set (Procedure 9.6), for which the QL-subpaths of $T$ consisting of singles give the QL-blocks of $(T, S)$; so, the maximal such subpaths of $T$ give all maximal QL-blocks of $(T, S)$.

Proposition 11.4. The upper horizontal of an anchor diagram $(T, S)$ of a direct QL-path $T=\left(X_{0}, \ldots, X_{n}\right)$ with $n \geq 2$ does not contain arrows $(\rightarrow)$ except those pointing to $X_{0}$ or $X_{n}$.

Proof. Suppose there is an arrow, say, $X_{p} \rightarrow X_{p+1}$ with $p+1<n$. Then $X_{p+2} \in \mathrm{QL}\left(X_{p+1}\right) \subset$ $\mathrm{QL}\left(X_{p}\right)$, and so the pair $\left(X_{p}, X_{p+2}\right)$ would be quasilinear, contradicting the assumption that $T$ is direct.

Definition 11.5. An arrow $X_{n-1} \rightarrow X_{n}$ gives us a diagram

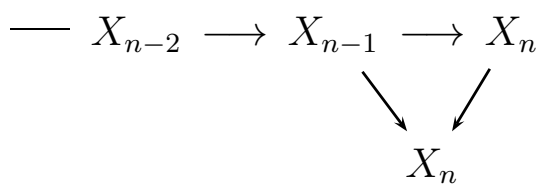

and thus $\left(X_{n-1}, X_{n}\right)$ is a twin pair. In the same way we obtain a twin pair $\left(X_{0}, X_{1}\right)$ from an arrow $X_{1} \rightarrow X_{0}$. We call these twin pairs $\left(X_{n-1}, X_{n}\right)$ and $\left(X_{0}, X_{1}\right)$ special twin pairs.

There are other possibilities to modify a minimal QL-path $\left(X_{0}, \ldots, X_{n}\right), n \geq 3$, to a path $\left(X_{0}, Y, X_{2}, \ldots, X_{n}\right)$ or $\left(X_{0}, \ldots, X_{n-2}, Y, X_{n}\right)$ which starts or ends with a twin pair $\left(X_{0}, Y\right)$ or $\left(Y, X_{n}\right)$. Recall from Scholium 14.17 that, given an optimal QL-path $\left(X_{0}, \ldots, X_{n}\right)$ and a ray $Y \in X_{0}^{\uparrow}$, the intersection $\mathrm{QL}(Y) \cap\left\{X_{0}, \ldots, X_{n}\right\}$ is contained in $\left\{X_{0}, X_{1}, X_{2}\right\}$. Since trivially $X_{1} \in \mathrm{QL}(Y)$, this intersection is either $\left\{X_{0}, X_{1}\right\}$ or $\left\{X_{0}, X_{1}, X_{2}\right\}$.

Definition 11.6. We say that the optimal $Q L$-path $\left(X_{0}, \ldots, X_{n}\right)$ has narrow entrance if $X_{2} \notin \mathrm{QL}(Y)$ for every $Y \in X_{0}^{\uparrow}$, and that $\left(X_{0}, \ldots, X_{n}\right)$ has wide entrance otherwise. Analogously we say that $\left(X_{0}, \ldots, X_{n}\right)$ has narrow exit, if $\mathrm{QL}(Y) \cap\left\{X_{0}, \ldots, X_{n}\right\}=\left\{X_{n-1}, X_{n}\right\}$ for every $Y \in X_{n}^{\uparrow}$, and wide exit otherwise. 
If $\left(X_{0}, \ldots, X_{n}\right)$ has narrow entrance, it can nevertheless happen that $X_{2} \in \mathrm{QL}(Y)$ for a ray $Y$ in $\left(X_{0}^{\downarrow}\right)^{\uparrow}$. In this case we have a diagram

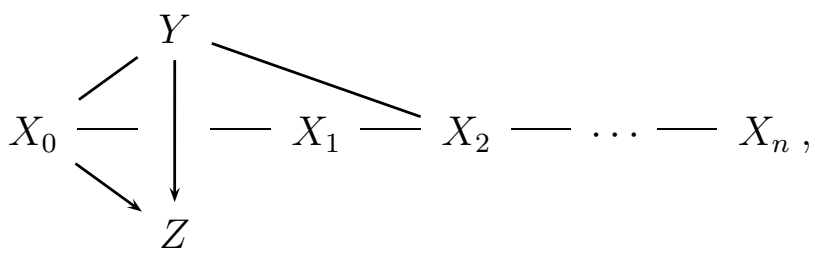

and so a new QL-path $T^{\prime}:=\left(X_{0}, Y, X_{2}, \ldots, X_{n}\right)$ of length $n$. We face the problem that $T^{\prime}$ perhaps is not a direct path. This problem vanishes if $T$ is minimal, since then $T^{\prime}$ is again minimal and so is direct. The following is now obvious.

Theorem 11.7. If $T=\left(X_{0}, \ldots, X_{n}\right)$ is a minimal QL-path with narrow entrance, and if $X_{2} \in \mathrm{QL}(Y)$ for a ray $Y$ in $\left(X_{0}^{\downarrow}\right)^{\uparrow}$, then the minimal $Q L$-path $T^{\prime}=\left(X_{0}, Y, X_{2}, \ldots, X_{n}\right)$ arising in the diagram (11.5) starts with a twin pair $\left(X_{0}, Y\right)$. Analogously, if $T$ has narrow exit and $W$ is a ray in $\left(X_{n}^{\downarrow}\right)^{\uparrow}$ with $X_{n-2} \in \mathrm{QL}(W)$, then $T^{\prime \prime}=\left(X_{0}, \ldots, X_{n-2}, W, X_{n}\right)$ is a minimal $Q L$-path ending with a twin pair $\left(W, X_{n}\right)$.

These modifications $T^{\prime}$ and $T^{\prime \prime}$ of $T$ have narrow entrance and narrow exit, respectively.

The following proposition indicates that minimal QL-paths with narrow entrance abound.

Proposition 11.8. Assume that $\left(X_{0}, \ldots, X_{n}\right)$ is a minimal $Q L$-path with $n>3$. Then for any $r \in[1, n-1]$ the subpath $\left(X_{r}, \ldots, X_{n}\right)$ is a minimal $Q L$-path with narrow entrance.

Proof. $\left\{X_{r}, \ldots, X_{n}\right\}$ is minimal, since $\left\{X_{0}, \ldots X_{n}\right\}$ is minimal. Let $Y \in X_{r}^{\uparrow}$. By Scholium 8.17

$$
\mathrm{QL}(Y) \cap\left\{X_{0}, \ldots, X_{n}\right\}=\left\{X_{r-1}, X_{r}, X_{r+1}\right\}
$$

and consequently $\mathrm{QL}(Y) \cap\left\{X_{r}, \ldots, X_{n}\right\}=\left\{X_{r}, X_{r+1}\right\}$.

If $T=\left(X_{0}, \ldots, X_{n}\right)$ is a minimal QL-path with wide entrance and $Y$ is a ray in $Z^{\uparrow}$ for some $Z \in X_{0}^{\downarrow}$ with $X_{2} \in \mathrm{QL}(Y)$, then $T^{\prime}=\left(X_{0}, Y, X_{2}, \ldots, X_{n}\right)$ is again a minimal QL-path which starts with a twin pair $\left(X_{0}, Y\right)$, as indicated in diagram (11.5). But now we already have a ray $W \in X_{0}^{\uparrow}$ at hands with $X_{2} \in \mathrm{QL}(W)$ and so a modification $\left(X_{0}, W, X_{2}, \ldots, X_{n}\right)=\widetilde{T}$ of $T$ which is a minimal QL-path starting with a twin-pair $\left(X_{0}, W\right)$. We meet a situation which indicates more freedom in the choice of minimal modifications of $T$ than in the case of narrow entrance. $X_{0}, W, Y$ are rays in the quasilinear convex set $Z^{\uparrow}$, and so every $Y^{\prime} \in[W, Y]$ is a ray with $\left(X_{0}, Y^{\prime}\right) \in Z^{\uparrow}$. Further all three pairs $(W, Y),\left(W, X_{2}\right),\left(Y, X_{2}\right)$ are quasilinear, and so the convex hull of $\left\{W, Y, X_{2}\right\}$ is quasilinear, which implies that $\left(Y^{\prime}, X_{2}\right)$ is quasilinear for every $Y^{\prime} \in[W, Y]$. Summarizing we obtain

Theorem 11.9. Assume that $T=\left(X_{0}, X_{1}, \ldots, X_{n}\right)$ is a minimal QL-path which has wide entrance, and so there exists a ray $W \in X_{0}^{\uparrow}$ with $X_{2} \in \mathrm{QL}(W)$. Assume further that there is given a ray $Y \in\left(X_{0}^{\downarrow}\right)^{\uparrow}$ with $X_{2} \in \mathrm{QL}(Y)$. Then for every $Y^{\prime} \in[W, Y]$ the sequence $\left(X_{0}, Y^{\prime}, X_{2}, \ldots, X_{n}\right)$ is a minimal QL-path, which starts with a twin pair $\left(X_{0}, Y^{\prime}\right)$ and has wide entrance. 


\section{Domination of minimal QL-Paths}

Recall that, given two QL-paths $T=\left(X_{0}, \ldots, X_{n}\right), T^{\prime}=\left(X_{0}^{\prime}, \ldots, X_{n}^{\prime}\right)$ of same length $n$, we say that $T^{\prime}$ dominates $T$, and write $T \preceq_{\mathrm{QL}} T^{\prime}$, if $X_{i} \preceq_{\mathrm{QL}} X_{i}^{\prime}$ for every $i$ in $[0, n]$ (Definition 8.19). In 88 we studied the domination relation when $T$ and $T^{\prime}$ are direct or optimal. When $T^{\prime}$ is minimal, we can say more.

Proposition 12.1. Let $T=\left(X_{0}, \ldots, X_{n}\right)$ and $T^{\prime}=\left(X_{0}^{\prime}, \ldots, X_{n}^{\prime}\right)$ be $Q L$-paths with $T \preceq_{\mathrm{QL}} T^{\prime}$. If $T^{\prime}$ is minimal, then $T$ is minimal.

Proof. Suppose there exists a QL-path $\left(X_{0}, Z_{1}, \ldots, Z_{r}, X_{n}\right)$ with $r<n-1$. Since QL $\left(X_{0}^{\prime}\right) \supset$ $\mathrm{QL}\left(X_{0}\right)$ and $\mathrm{QL}\left(X_{n}^{\prime}\right) \supset \mathrm{QL}\left(X_{n}\right)$, the pairs $\left(X_{0}^{\prime}, Z_{1}\right)$ and $\left(Z_{r}, X_{n}^{\prime}\right)$ are quasilinear, and so $\left(X_{0}^{\prime}, Z_{1}, \ldots, Z_{r}, X_{n}^{\prime}\right)$ is a QL-path of length $<n$, contradicting the minimality of $T^{\prime}$. Thus $T$ is minimal.

Given a minimal QL-path $T=\left(X_{0}, \ldots, X_{n}\right)$ and a QL-path $T^{\prime}=\left(X_{0}^{\prime}, \ldots, X_{n}^{\prime}\right)$ with $T \preceq_{\mathrm{QL}} T^{\prime}$, it is trivial that $T^{\prime}$ is also minimal in the case that $X_{0}^{\prime}=X_{0}$ and $X_{n}^{\prime}=X_{n}$, simply since $T^{\prime}$ has length $n$. More interest deserves to dominate a subpath of $T$ by a minimal QL-path as follows.

Proposition 12.2. Assume that $T=\left(X_{0}, \ldots, X_{n}\right)$ is a minimal $Q L$-path and $\left(X_{1}^{\prime}, \ldots, X_{n-1}^{\prime}\right)$ is a $Q L$-path (of length $n-2$ ) dominating the subpath $\left(X_{1}, \ldots, X_{n-1}\right)$ of T. Then $\left(X_{1}^{\prime}, \ldots, X_{n-1}^{\prime}\right)$ is minimal.

Proof. The pairs $\left(X_{0}, X_{1}^{\prime}\right)$ and $\left(X_{n}, X_{n-1}^{\prime}\right)$ are quasilinear, since $\mathrm{QL}\left(X_{1}^{\prime}\right) \supset \mathrm{QL}\left(X_{1}\right)$ and $\mathrm{QL}\left(X_{n-1}^{\prime}\right) \supset \mathrm{QL}\left(X_{n-1}\right)$. Thus $T^{\prime}:=\left(X_{0}, X_{1}^{\prime}, \ldots, X_{n-1}^{\prime}, X_{n}\right)$ is a QL-path of length $n$ from $X_{0}$ to $X_{n}$, and so is minimal. The subpath $\left(X_{1}^{\prime}, \ldots, X_{n-1}^{\prime}\right)$ of $T^{\prime}$ is again minimal.

Remark 12.3. Proposition 12.2 gives us a $Q L$-path $T_{0}=\left(X_{1}, \ldots, X_{n-1}\right)$ such that every QL-path dominating $T_{0}$ is minimal. Thus $T_{0}$ is an optimal path, all of whose enlargements are again optimal.

Theorem 12.4. Assume that $T=\left(X_{0}, \ldots, X_{n}\right)$ and $T^{\prime}=\left(X_{0}^{\prime}, \ldots, X_{n}^{\prime}\right)$ are minimal $Q L$ paths with $T \preceq_{\mathrm{QL}} T^{\prime}$ and that $S=\left(Y_{0}, \ldots, Y_{m}\right)$ is an anchor set of $T$.

a) Then $S$ is also an anchor set of $T^{\prime}$.

b) If $p \in[0, n-1]$ then $\left(X_{p}, X_{p+1}\right)$ is a twin pair in $T$ iff $\left(X_{p}^{\prime}, X_{p+1}^{\prime}\right)$ is a twin pair in $T^{\prime}$.

c) If $p \in[0, n]$ then $X_{p}$ is a single in $T$ iff $X_{p}^{\prime}$ is a single in $T^{\prime}$.

d) For each $p \in[0, n]$ have $X_{p}$ and $X_{p}^{\prime}$ the same legal anchor and the same illegal anchors (if any) in $S$.

Proof. For every $i \in[0, n]$ we have the (minimal) subpaths $\left(X_{0}, \ldots, X_{i}\right)$ and $\left(X_{0}^{\prime}, \ldots, X_{i}^{\prime}\right)$ of $T$ and $T^{\prime}$ with $\left(X_{0}, \ldots, X_{i}\right) \preceq_{\mathrm{QL}}\left(X_{0}^{\prime}, \ldots, X_{i}^{\prime}\right)$ and an anchor set $\left(Y_{0}, \ldots, Y_{m(i)}\right)$ of $\left(X_{0}, \ldots X_{i}\right)$ with $0=m(0) \leq m(1) \leq \cdots \leq m(n)=m$. The claims a) $-\mathrm{d}$ ) can be verified successively for these sequences in a straightforward way by looking at Definitions 9.5 and 10.6. This proves the theorem.

Corollary 12.5. In the situation of Theorem 12.4 also the following holds.

a) If $0 \leq p<p+t \leq n$, then $\left(X_{p}, \ldots, X_{p+t}\right)$ is a flock in $T$ iff $\left(X_{p}^{\prime}, \ldots, X_{p+t}^{\prime}\right)$ is a flock in $T^{\prime}$. 
b) A twin pair $\left(X_{p}, X_{p+1}\right)$ in $T$ is isolated in $T$ iff $\left(X_{p}^{\prime}, X_{p+1}^{\prime}\right)$ is isolated in $T^{\prime}$.

c) The QL-path $T^{\prime}$ is flocky iff $T$ is flocky.

Proof. Claims a) and b) are evident by Theorem 15.4, since flocks and isolated twin pairs can be characterized in terms of the anchors in $S$ of the involved rays, as made explicit in Scholium 10.7. The same holds for the property "flocky", namely $T$ is flocky iff for any $k \in[0, m-1]$ with $Y_{k}^{\uparrow} \cap Y_{k+1}^{\uparrow}$ not empty there is a ray $X_{p}$ in $T$ which has the legal anchor $Y_{k}$ and the illegal anchor $Y_{k+1}$, and this happens iff $X_{p}^{\prime}$ has these legal and illegal anchors.

We are aware that a large part of the contents of Theorem 12.4 and Corollary 12.5 can be proved without employing our elaborate theory of anchors. In particular this holds for assertions b) and c) in Theorem 12.4.

\section{REFERENCES}

[1] M. Akian, S. Gaubert, and A. Guterman. Linear independence over tropical semirings and beyond. In Tropical and Idempotent Mathematics, G.L. Litvinov and S.N. Sergeev, (eds.), Contemp. Math., 495:1-38, 2009.

[2] J. Golan. Semirings and their Applications, Springer-Science + Business, Dordrecht, 1999. (Originally published by Kluwer Acad. Publ., 1999.)

[3] Z. Izhakian. Tropical arithmetic and matrix algebra. Comm. in Algebra, 37(4):1445-1468, 2009.

[4] Z. Izhakian, M. Knebusch, and L. Rowen. Supertropical linear algebra. Pacific J. of Math., 266(1):43$75,2013$.

[5] Z. Izhakian, M. Knebusch, and L. Rowen. Supertropical quadratic froms I, J. of Pure and App. Algebra, 220(1):61-93, 2016.

[6] Z. Izhakian, M. Knebusch, and L. Rowen. Supertropical Quadratic Forms II: Tropical trigonometry and applications, Inter. J. of Alg. and Comput., to appear.

[7] Z. Izhakian, M. Knebusch, and L. Rowen. Minimal orderings and quadratic forms on a free module over a supertropical semiring, Linear Algebra and its Applications, 507:420-461, 2016.

[8] Z. Izhakian, M. Knebusch, and L. Rowen. Quadratic and symmetric bilinear forms on modules with unique base over a semiring, Documenta Mathematica, 21:773-808, 2016.

[9] Z. Izhakian, M. Knebusch, and L. Rowen. Supertropical semirings and supervaluations. J. Pure and Appl. Alg., 215(10):2431-2463, 2011.

[10] Z. Izhakian, M. Knebusch, and L. Rowen. Decompositions of modules lacking zero sums. Israel J. Math., 225(2):503-524, 2018.

[11] Z. Izhakian and L. Rowen. Supertropical algebra. Adv. in Math., 225(4):2222-2286, 2010.

[12] Z. Izhakian, L. Rowen. The tropical rank of a tropical matrix. Commun. Alg., 37(11):3912-3927, 2009.

[13] Z. Izhakian, L. Rowen. Supertropical matrix algebra. Israel J. Math., 182(1):383-424, 2011.

[14] Z. Izhakian, L. Rowen. Supertropical matrix algebra II: Solving tropical equations. Israel J. Math., 186(1):69-97, 2011.

[15] Z. Izhakian, L. Rowen. Supertropical matrix algebra III: Powers of matrices and generalized eigenspaces. J. Alg., 341(1):125-149, 2011.

[16] M. Knebusch and D. Zhang. Convexity, valuations, and Prüfer extensions in real algebra, Doc. Math., 10:1-109, 2005.

Institute of Mathematics, University of Aberdeen, AB24 3UE, Aberdeen, UK.

E-mail address: zzur@abdn.ac.uk

Department of Mathematics, NWF-I Mathematik, Universität Regensburg 93040 RegensBURG, Germany

E-mail address: manfred.knebusch@mathematik.uni-regensburg.de 